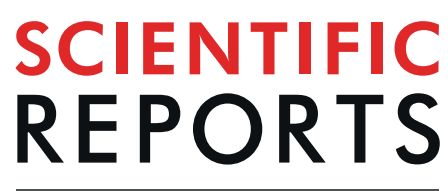

\title{
Neuroestrogen synthesis modifies neural representations of learned song without altering vocal imitation in developing songbirds
}

Daniel M. Vahaba $\mathbb{1}^{1,2^{*}}$, Amelia Hecsh ${ }^{3}$ \& Luke Remage-Healey $\mathbb{1}^{2,3,4^{*}}$

Birdsong learning, like human speech, depends on the early memorization of auditory models, yet how initial auditory experiences are formed and consolidated is unclear. In songbirds, a putative cortical locus is the caudomedial nidopallium (NCM), and one mechanism to facilitate auditory consolidation is $17 \beta$-estradiol (E2), which is associated with human speech-language development, and is abundant in both NCM and human temporal cortex. Circulating and NCM E2 levels are dynamic during learning, suggesting E2's involvement in encoding recent auditory experiences. Therefore, we tested this hypothesis in juvenile male songbirds using a comprehensive assessment of neuroanatomy, behavior, and neurophysiology. First, we found that brain aromatase expression, and thus the capacity to synthesize neuroestrogens, remains high in the auditory cortex throughout development. Further, while systemic estrogen synthesis blockade suppressed juvenile song production, neither systemic nor unilateral E2 synthesis inhibition in NCM disrupted eventual song imitation. Surprisingly, early life neuroestrogen synthesis blockade in NCM enhanced the neural representations of both the birds' own song and the tutor song in NCM and a downstream sensorimotor region, HVC, respectively. Taken together, these findings indicate that E2 plays a multifaceted role during development, and that, contrary to prediction, tutor song memorization is unimpaired by unilateral estrogen synthesis blockade in the auditory cortex.

While many animals use sounds to communicate with one another (vocal communication), the ability to learn to vocally communicate is relatively rare ${ }^{1}$. In vocal learning animals, such as humans and songbirds, vocal learning occurs across two main phases: an auditory memorization ('sensory') phase, followed by a sensorimotor phase ('babbling, error correction/feedback) ${ }^{2,3}$. While much is known about sensorimotor learning, the mechanisms that guide the formation and consolidation of auditory memories early in life are less clear.

One brain region likely involved in storing auditory memories in songbirds is the caudomedial nidopallium $(\mathrm{NCM})^{4}$. NCM, comparable to mammalian secondary auditory cortex, is required for accurate song learning. Blocking ERK-signaling or mTOR bilaterally in NCM during tutoring leads to poor song imitation ${ }^{5,6}$. Tutoring naïve juvenile songbirds leads to an increased proportion of tutor-song-selective neurons in $\mathrm{NCM}^{7}$. Further, bilateral NCM lesions abolish innate preference for tutor song in adults ${ }^{8}$, but see ${ }^{9,10}$. Thus, NCM contains a putative tutor 'engram'; however, the neuromodulatory mechanisms that shape this auditory memory formation and consolidation remain unknown.

Consolidating recent experience in other contexts and systems require presynaptic signaling molecules ('neuromodulators'), such as brain-derived estrogens ${ }^{11}$. The predominant estrogen $17 \beta$-estradiol (E2) is a candidate neuromodulator required for auditory memory consolidation, as is evident in a similar role in adult hippocampal-dependent cognition, across taxa ${ }^{12-28}$, but $\operatorname{se}^{29}$. Additionally, both circulating and brain-derived estrogens ('neuroestrogens') typically enhance hearing in non-human animals ${ }^{30,31}$, and estrogens are associated

${ }^{1}$ Department of Biological Sciences, Program in Neuroscience, Smith College, Northampton, 01063, USA. ${ }^{2}$ Center for Neuroendocrine Studies, University of Massachusetts Amherst, Amherst, MA, 01002, USA. ${ }^{3}$ Department of Psychological \& Brain Sciences, University of Massachusetts Amherst, Amherst, MA, 01002, USA. ${ }^{\text {Neuroscience }}$ \& Behavior Graduate Program, Center for Neuroendocrine Studies, Department of Psychological \& Brain Sciences, University of Massachusetts Amherst, Amherst, MA, 01002, USA. *email: dvahaba@smith.edu; healey@cns.umass.edu 


\begin{tabular}{|c|c|c|c|c|c|c|c|c|}
\hline Phase & Subregion & Hemisphere & Subjects & Images & Aromatase & Parvalbumin & $\begin{array}{l}\text { Aromatase- } \\
\text { Parvalbumin }\end{array}$ & DAPI \\
\hline \multirow{4}{*}{ Sensory } & \multirow{2}{*}{$\mathrm{dNCM}$} & $\mathrm{L}$ & 2 & 3 & $153.45 \pm 8.22$ & $15.74 \pm 3.55$ & $14.26 \pm 6.16$ & $572.50 \pm 58.36$ \\
\hline & & $\mathrm{R}$ & 3 & 4 & $159.36 \pm 14.34$ & $13.65 \pm 1.74$ & $9.59 \pm 3.04$ & $553.32 \pm 24.90$ \\
\hline & \multirow{2}{*}{ vNCM } & $\mathrm{L}$ & 2 & 3 & $139.68 \pm 5.48$ & $14.26 \pm 1.30$ & $21.15 \pm 7.09$ & $467.25 \pm 86.08$ \\
\hline & & $\mathrm{R}$ & 3 & 5 & $146.08 \pm 14.10$ & $17.71 \pm 2.09$ & $10.33 \pm 4.64$ & $455.64 \pm 44.55$ \\
\hline \multirow{4}{*}{ Sensorimotor } & \multirow{2}{*}{ dNCM } & $\mathrm{L}$ & 3 & 6 & $139.93 \pm 13.44$ & $17.46 \pm 2.10$ & $10.33 \pm 2.98$ & $467.74 \pm 14.49$ \\
\hline & & $\mathrm{R}$ & 2 & 4 & $149.03 \pm 16.82$ & $15.86 \pm 1.52$ & $15.86 \pm 5.12$ & $469.22 \pm 50.22$ \\
\hline & \multirow{2}{*}{ vNCM } & $\mathrm{L}$ & 3 & 6 & $119.76 \pm 12.44$ & $14.26 \pm 2.31$ & $10.08 \pm 1.92$ & $418.80 \pm 28.69$ \\
\hline & & $\mathrm{R}$ & 2 & 4 & $135.38 \pm 21.69$ & $18.44 \pm 2.29$ & $10.33 \pm 4.39$ & $393.96 \pm 44.88$ \\
\hline
\end{tabular}

Table 1. Density measures for antibody staining in developing NCM. Values represent mean cell density $\left(\mathrm{mm}^{3}\right)$ $+/-$ the standard error of the mean.

with language and verbal memory in humans ${ }^{32-36}$. Together, current evidence suggests that neuroestrogen signaling may facilitate the consolidation of recent auditory experience ${ }^{37}$.

Neuroestrogens rapidly enhance the gain and coding of auditory neurons in NCM across the lifespan ${ }^{38-40}$. Thus, it is possible that one functional role of E2 acting within the auditory forebrain is to facilitate song memory consolidation. NCM is uniquely enriched with estrogen synthase (aromatase) in vocal learning birds ${ }^{41}$, suggesting its presence is distinctly important for song learning. Further, systemic inhibition of estrogen synthesis during training and testing results in impaired auditory recognition in adult zebra finches ${ }^{42}$. However, it is unclear how neuroestrogens may affect tutor song memorization during and/or after a song learning experience. Currently, there are several pieces of evidence that suggest a functional role for E2 during the vocal learning critical period. In songbirds, circulating E2 levels rise during the sensory phase, and at least in swamp sparrows, predict future tutor imitation success ${ }^{43-46}$, as in humans with language ${ }^{35,47}$. Moreover, the expression of GPER1 (a membrane-bound estrogen receptor proposed to mediate the rapid effects of E2) peaks in the telencephalon of male songbirds during the sensory phase ${ }^{48}$. As with E2-dependent learning in rodents, E2 levels are rapidly elevated in NCM immediately after a song learning session ${ }^{49}$.

The aim of the present study was to determine whether E2 synthesis supports the consolidation of a recent auditory experience and the eventual vocal imitation of a tutor model. Based on prior findings, we postulated that elevated E2 levels in the auditory forebrain aid in memory consolidation following individual song learning bouts. We tested whether the eventual degree of vocal similarity between the social model (tutor) and the pupil in adulthood would be impaired by inhibiting neuroestrogen synthesis in NCM during and immediately after bouts of vocal communication learning.

\section{Results}

Cell density is region- and age-dependent in developing auditory forebrain while aromatase and parvalbumin expression are unchanging. We first sought to confirm the presence of aromatase in NCM across development. While previous studies have characterized aromatase protein ${ }^{50}$ and $\mathrm{mRNA}^{49,51-53}$ expression in the brains of developing songbirds, aromatase protein expression specifically within NCM between the sensory and sensorimotor phase of the song learning period has not been assessed, to our knowledge. In addition to aromatase, we were also curious as to whether transitions between learning phases were associated with differences in expression of the calcium buffering-protein parvalbumin (PV). Parvalbumin is a marker for a unique subpopulation of inhibitory interneurons ${ }^{54}$, is co-localized with aromatase in $\mathrm{NCM}^{55}$, and its presence often denotes changes in critical period plasticity within mammalian visual cortex ${ }^{56}$, as well as songbird song circuits $^{57}$. Further, E2 may modulate auditory circuits by modulating inhibitory interneurons such as PV + cells $^{37}$. We focused solely on males as they were the sex of interest for subsequent song and physiology experiments in this study. Although we collected both hemispheres of NCM for this experiment, we excluded hemisphere as a factor in our statistical model to support sufficient statistical power for our comparisons. Qualitatively, we found similar expression of aromatase and parvalbumin across both hemispheres of sensory- and sensorimotor-aged subjects (see Tables 1 and 2).

We divided our subjects into two age groups reflecting the two different developmental song learning phases: sensory- and sensorimotor-aged (20-34 and 65-71 dph, respectively $\left.{ }^{40}\right)$. Overall, density measures revealed comparable aromatase expression, parvalbumin expression, and aromatase-parvalbumin co-expression in both dorsal and ventral NCM across development (aromatase: $F_{(1,31)}=2.458, p=0.127$; parvalbumin: $F_{(1,31)}=0.035$, $p=0.854$; aromatase-parvalbumin: $\left.F_{(1,31)}=0.003, p=0.957\right)$, age (aromatase: $F_{(1,31)}=2.218, p=0.147$; parvalbu$\min : F_{(1,31)}=0.277, p=0.602$; aromatase-parvalbumin: $\left.F_{(1,31)}=0.339, p=0.565\right)$, without any significant interactions between age and region (aromatase: $F_{(1,31)}=0.048$; parvalbumin: $F_{(1,31)}=0.751$; aromatase-parvalbumin: $F_{(1,31)}=0.757 ; p>0.3$ for all tests; Table 1$)$.

Interestingly, we observed a significantly higher total number of cells (DAPI-positive nuclei) in dorsal NCM compared to ventral NCM $\left(F_{(1,31)}=8.128, p=0.008\right)$, and in sensory-aged animals compared to sensorimotor-aged subjects $\left(F_{(1,31)}=6.291, p=0.018\right.$; Fig. 1C,D $)$. No significant interactions emerged between region and age $\left(F_{(1,31)}=0.587, p=0.449\right)$. There were no differences between age and NCM subregion when we normalized the markers of interest (aromatase and parvalbumin) to the relative amount of DAPI to account for subject and image variability (Fig. 1A,B; see Tables 1 and 2 for all descriptive data for density and \% of DAPI 


\begin{tabular}{|c|c|c|c|c|c|c|c|}
\hline Phase & Subregion & Hemisphere & Subjects & Images & Aromatase & Parvalbumin & $\begin{array}{l}\text { Aromatase- } \\
\text { Parvalbumin }\end{array}$ \\
\hline \multirow{4}{*}{ Sensory } & \multirow{2}{*}{$\mathrm{dNCM}$} & $\mathrm{L}$ & 2 & 3 & $27.09 \pm 1.43$ & $2.79 \pm 0.66$ & $2.50 \pm 1.06$ \\
\hline & & $\mathrm{R}$ & 3 & 4 & $29.23 \pm 3.61$ & $2.48 \pm 0.34$ & $1.71 \pm 0.48$ \\
\hline & \multirow{2}{*}{ vNCM } & $\mathrm{L}$ & 2 & 3 & $32.09 \pm 6.19$ & $3.18 \pm 0.35$ & $4.21 \pm 0.90$ \\
\hline & & $\mathrm{R}$ & 3 & 5 & $32.69 \pm 2.68$ & $4.08 \pm 0.67$ & $2.18 \pm 1.00$ \\
\hline \multirow{4}{*}{ Sensorimotor } & \multirow{2}{*}{$\mathrm{dNCM}$} & L & 3 & 6 & $29.86 \pm 2.48$ & $3.70 \pm 0.35$ & $2.22 \pm 0.63$ \\
\hline & & $\mathrm{R}$ & 2 & 4 & $32.21 \pm 2.96$ & $3.53 \pm 0.57$ & $3.13 \pm 0.78$ \\
\hline & \multirow{2}{*}{ vNCM } & $\mathrm{L}$ & 3 & 6 & $28.28 \pm 1.43$ & $3.47 \pm 0.55$ & $2.46 \pm 0.55$ \\
\hline & & $\mathrm{R}$ & 2 & 4 & $35.02 \pm 5.26$ & $4.73 \pm 0.39$ & $2.40 \pm 0.98$ \\
\hline
\end{tabular}

Table 2. Protein expression relative to cell density (\% DAPI). Values represent mean number of immunopositive-cells relative to the number of DAPI+ neurons (\% DAPI) $+/$ - the standard error of the mean.

A.

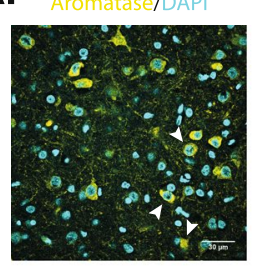

Parvalbumin/DAP!

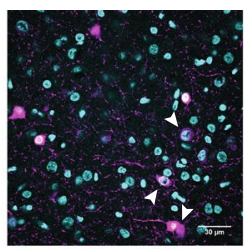

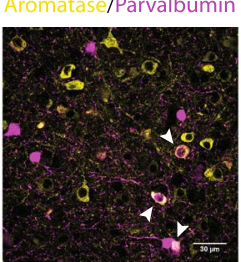
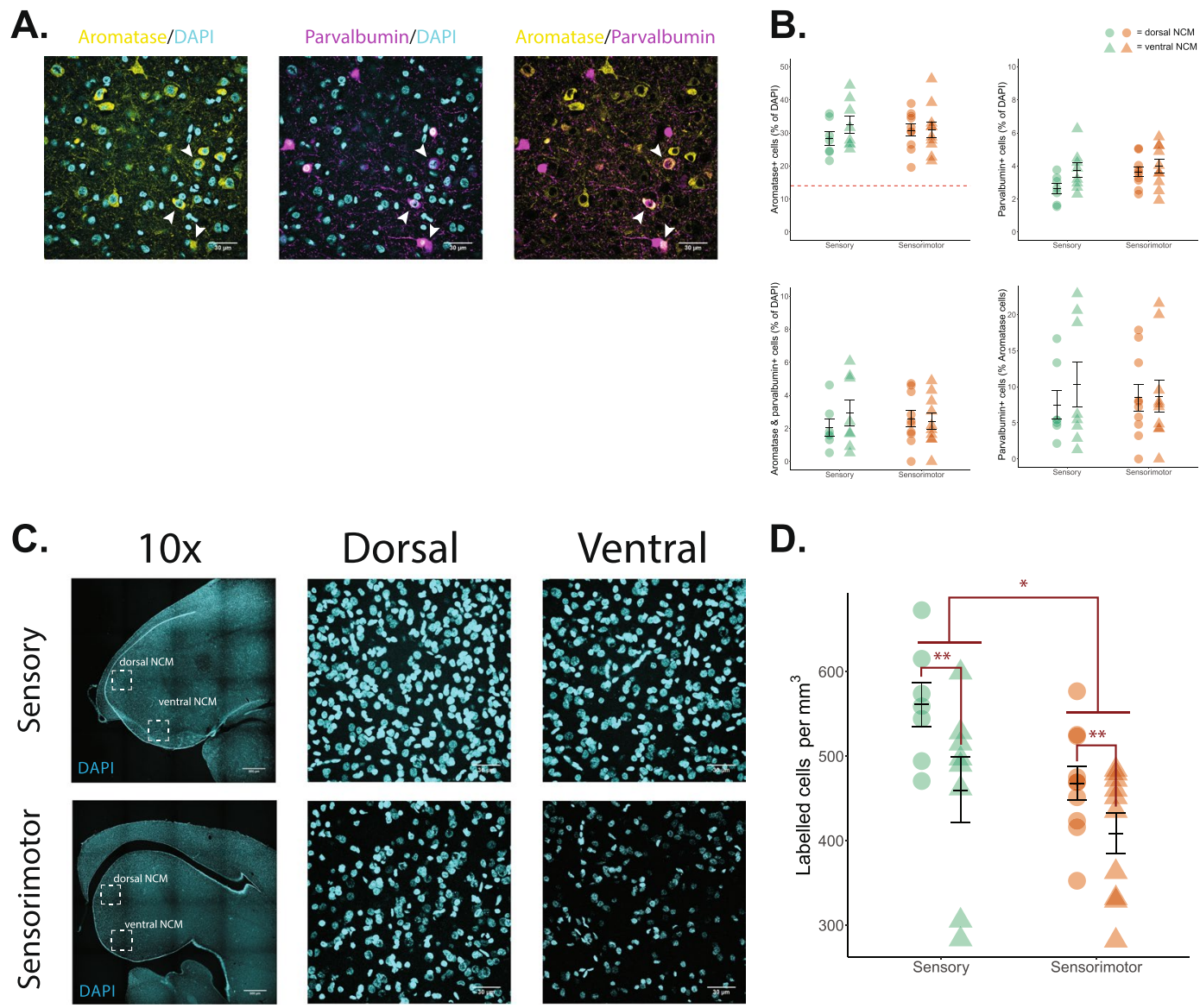

D.

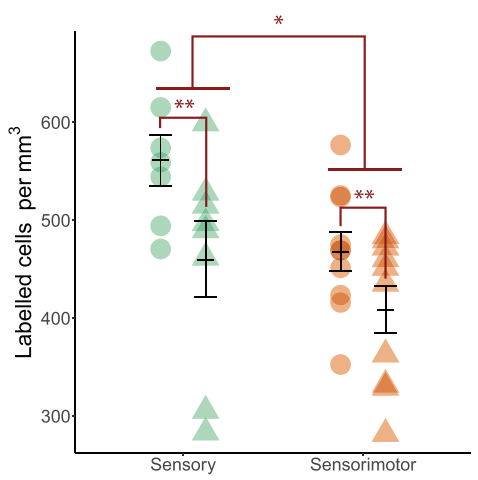

Figure 1. Changes in neuronal density and aromatase and parvalbumin expression in NCM across development. (A) Aromatase, parvalbumin, aromatase parvalbumin co-expression, respectively, from an exemplar sensory-aged male bird (26 dph; right hemisphere; ventral NCM). Pseudo-colored: yellow, aromatase; cyan, DAPI; magenta, parvalbumin. Each image from a single slice of a z-stack taken at 60x magnification. Scale bar $=30 \mu \mathrm{m}$. White arrowheads indicate aromatase and parvalbumin co-expression. (B) Expression of aromatase, parvalbumin, and aromatase/parvalbumin co-expression, respectively, relative to the number of DAPI-positive nuclei (\%), and parvalbumin co-expression relative to total aromatase expression (\%). Overall, there are no significant differences in expression by age or NCM subregion. Circles = dorsal NCM; triangles $=$ ventral NCM; green $=$ sensory-aged birds; orange $=$ sensorimotor-aged birds. $(\mathbf{C})$ Total cell counts (DAPI-positive nuclei) across development; top row: sensory-aged bird ( $25 \mathrm{dph}$; right NCM); bottom row: sensorimotor-aged bird (71 dph; right NCM). 10x images taken from a $4 \times 4$ stitched image. Dorsal and ventral NCM images taken from a z-project max intensity 60x image. Note, only 60x images were quantified. (D), Cell density (total cell counts; DAPI-positive nuclei) by region and age. Dorsal NCM shows higher cell density than ventral NCM. Similarly, sensory-aged birds have higher overall cell density across subregions compared to sensorimotor-aged subjects. ${ }^{*} p<0.05 ; * p<0.001$. 


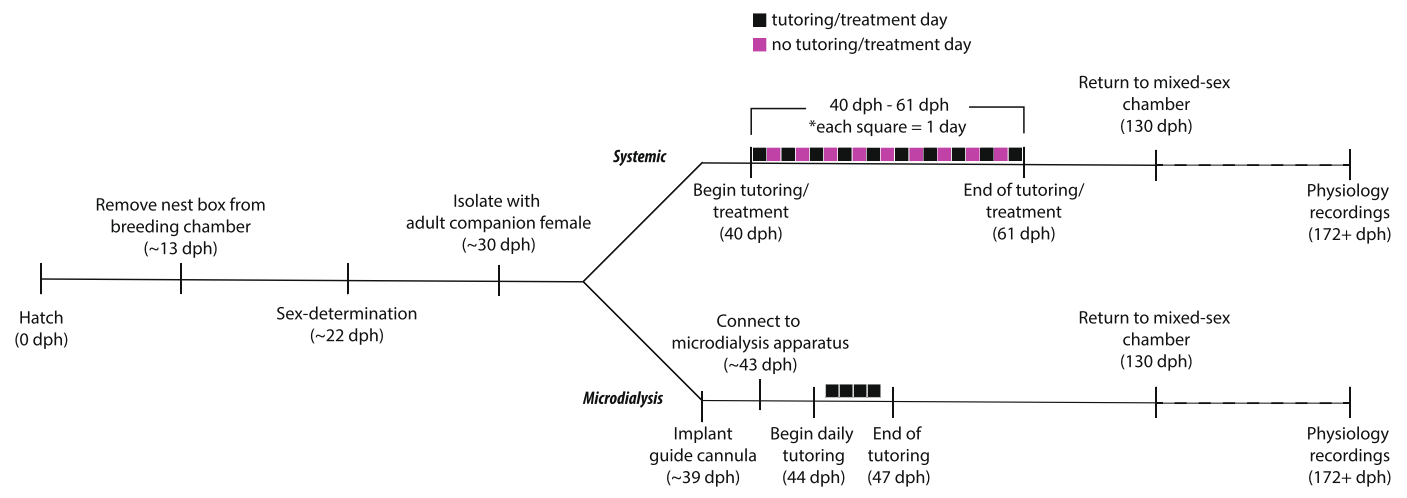

Figure 2. Experimental timeline.

measurements). Overall, these findings confirm that aromatase and parvalbumin are present in the developing auditory forebrain, and suggests that NCM may experience apoptosis as birds develop while maintaining subregion differences in cell density.

Song learning is unaffected by global estrogen synthesis inhibition during development. Systemic administration. Birds in this experiment received an oral administration of either FAD or saline every other day for 20 days immediately following tutoring (Fig. 2). This tutoring/treatment regimen allowed us to examine E2's role specifically in post-training memory consolidation instead of the well-studied effect of E2 during auditory processing (see Introduction). Systemic FAD suppresses circulating E2 levels for at least 48 hours $^{58}$, whereas our goal was to test E2's role specifically in the post-auditory experience phase. Initially, we measured the daily amount of song systemically-treated animals produced before $(<40 \mathrm{dph})$ and during the tutoring period (40-60 dph) as global inhibition of estrogen synthesis in adult songbirds can reduce song production ${ }^{59}$. Pre-tutoring, birds produced similar amounts of sang independent of the time of day or future treatment group (treatment: $F_{(1,13)}=2.466, p=0.140$; time of day: $F_{(1,13)}=1.797, p=0.203$; treatment $*$ time of day: $F_{(1,13)}=0.719$, $p=0.412$; Fig. $3 A, B)$. However, during the tutoring period, FAD treatment significantly suppressed song production $\left(\mathrm{FAD}=63.8 \pm 13.6\right.$ bouts; saline $=116.0 \pm 14.4$ bouts; $F_{(1,103)}=6.623, p=0.012$; Tukey's HSD: $\left.p=0.012\right)$ independent of time of day $\left(F_{(1,103)}=0.222, p=0.639\right)$ or an interaction between time of day and treatment $\left(F_{(1,}\right.$ ${ }_{103)}=1.882, p=0.173$; Fig. $\left.3 \mathrm{~A}, \mathrm{~B}\right)$. Interestingly, while initial song production was reduced during development, eventual song similarity at $130 \mathrm{dph}$ (one-way ANOVA (treatment); $F_{(1,4)}=0.064$ ), accuracy $\left(F_{(1,4)}=0.021\right)$, and sequential similarity $\left(F_{(1,4)}=0.095\right)$ were statistically similar when both FAD and saline subjects reached adulthood ( $p>0.77$; Fig. 3D \& Table 3). Additionally, there was no effect of treatment $\left(F_{(1,4)}=0.427, p=0.549\right)$, nor an interaction of treatment with age $\left(\mathrm{F}_{(4,16)}=0.569, p=0.689\right)$. There was, however, a significant increase in song similarity as birds reached adulthood (age: $F_{(4,16)}=5.528, p=0.005$; post-hocs: $p<0.05$ for 49 dph vs. $86 \& 130$ $\mathrm{dph}$; all other age comparisons non-significant, $p>0.06$; Fig. $3 \mathrm{C}$ ). Together, these data show that systemic estrogen synthesis can support song production during the juvenile learning period, but it does not impact eventual tutor song imitation.

Developmental changes (relative to pre-tutoring values) in Wiener entropy (WE) and entropy variance (EV) during tutoring predict adult tutor song fidelity ${ }^{60}$. Independent of treatment, we tested this relationship for birds in the present experiment to assess whether they developed along a 'typical' song learning trajectory. In agreement with the previous report, we found a strong, significant positive correlation between change in WE at 49 $\mathrm{dph}$ (the first day all subjects produced song post-tutoring) and percent song similarity in adulthood (130dph); $r(4)=-0.951, p=0.004$, as well as a similar significant correlation when we considered entropy variance instead of WE $(r(4)=0.863, p=0.027$; Fig. 3E). Therefore, systemic FAD treatment did not impact song learning, and developing song was predictive of eventual similarity, indicating that our daily treatment regimen did not impair a 'normal' song learning trajectory.

Female phonotaxis behavior. While song similarity data can provide information on how well a bird imitates a model song, there may be subtle song features affected by early-life manipulations that are not captured by automated analyses. As adult female zebra finches use courtship song to evaluate potential life-long mates ${ }^{61}$, we asked whether a females' phonotactic response to song was impacted by a males' treatment during development. We found a significant interaction between treatment and trial day $\left(F_{(1,17)}=7.30, p=0.151\right)$. Follow-up analyses revealed that on the first day of phonotaxis, females spent more time near the speaker broadcasting a FAD-treated bird's song $(p=0.015)$, whereas on the second day there was nonsignificant tendency to spend more time near a control bird's song $(p=0.059$; Supp. Fig. 1A,B). Thus, we find no evidence that estrogen synthesis blockade influenced the female phonotaxis for song playback.

Song learning is unaffected by inhibition of local estrogen synthesis in NCM during development. In vivo microdialysis with social + playback tutoring. Systemic treatments yielded no effect of aromatase blockade, but leaves open the possibility that temporally-precise, site-directed manipulations within NCM could impact auditory memorization. However, as with systemically-administered subjects, unilateral FAD treatment in NCM did not modify eventual tutor imitation, nor did the cannulated hemisphere or interaction between treatment and hemisphere affect percent similarity $\left(F_{(1,16)}\right.$, treatment $=0.965$; hemisphere $=0.056$; 
A.

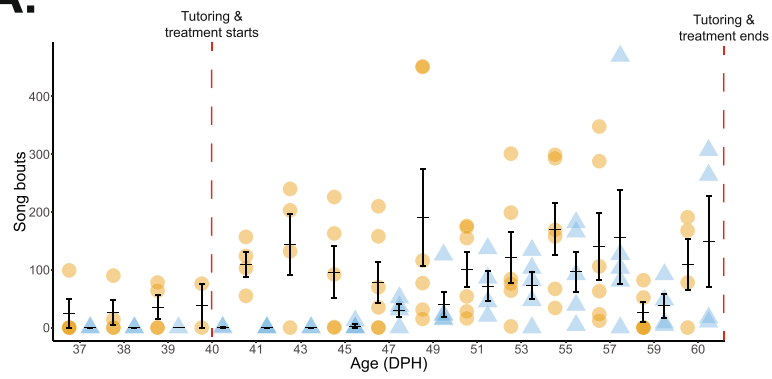

D.

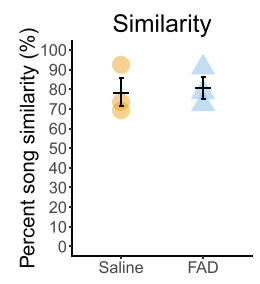

B.

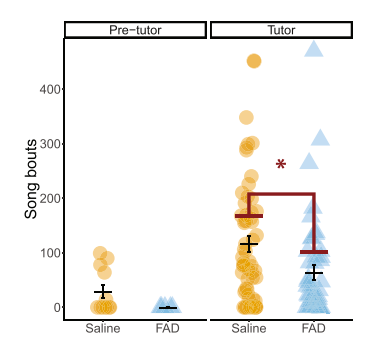

E.



C.

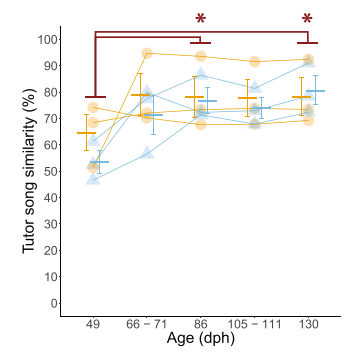

Figure 3. Systemic estrogen synthesis inhibition suppresses song production without impacting tutor song copying. (A) Daily number of song bouts before and across the tutoring/treatment period. (B) Birds sing amounts before treatment/tutoring; however, systemic FAD treatment reduces song production $(p=0.012)$. Circles/orange $=$ saline-treated birds $(n=3)$; triangles/blue $=$ FAD birds $(n=3) .(\mathbf{C})$ Song similarity is lowest at $49 \mathrm{dph}$ despite treatment (effect of age: $p=0.005$; * is relative to $49 \mathrm{dph}$ ). (D) At $130 \mathrm{dph}$, tutor song similarity, accuracy, and sequence similarity, respectively, are all similar across treatments. (E) Change in Wiener entropy at $49 \mathrm{dph}$ (post-tutoring day \#5) relative to pre-tutoring values predicts eventual percent song similarity to the tutor at $130 \mathrm{dph}$, independent of treatment $\left(r^{2}=0.903 ; p=0.004\right) . * p<0.05$.

\begin{tabular}{|l|l|l|l|l|l|l|}
\hline \multirow{3}{*}{ Method } & Treatment & Hemisphere & Subjects & Similarity & Accuracy & $\begin{array}{l}\text { Sequential } \\
\text { match }\end{array}$ \\
\hline \multirow{3}{*}{ Systemic } & Saline & - & 3 & $78.38 \pm 7.13$ & $77.52 \pm 3.52$ & $56.01 \pm 7.28$ \\
\cline { 2 - 7 } & FAD & - & 3 & $80.66 \pm 5.50$ & $78.04 \pm 0.79$ & $58.64 \pm 4.47$ \\
\hline \multirow{5}{*}{ Microdialysis } & \multirow{2}{*}{ aCSF } & Feft & 5 & $60.71 \pm 9.19$ & $74.40 \pm 2.07$ & $60.57 \pm 3.04$ \\
\cline { 3 - 7 } & \multirow{2}{*}{ FAD } & Reft & 5 & $63.51 \pm 7.53$ & $71.69 \pm 2.31$ & $64.17 \pm 4.47$ \\
\cline { 2 - 7 } & \multirow{2}{*}{ Cannula } & Right & 5 & $52.79 \pm 9.22$ & $75.74 \pm 1.07$ & $68.20 \pm 9.04$ \\
\cline { 2 - 7 } & Right & 4 & $54.15 \pm 9.13$ & $74.43 \pm 1.37$ & $69.99 \pm 8.91$ \\
\cline { 2 - 7 } & Isolate & Left & 1 & $24.56 \pm-$ & $68.40 \pm-$ & $85.01 \pm-$ \\
\hline
\end{tabular}

Table 3. Automated song similarity measurements. Values represent mean $+/-$ the standard error of the mean for each song similarity metric.

treatment $*$ hemisphere $=0.007 ; p>0.340)$, accuracy $\left(F_{(1,16)}\right.$, treatment $=1.325$; hemisphere $=1.277$; treatment $*$ hemisphere $=0.157 ; p>0.266)$, or sequence similarity $\left(F_{(1,16)}\right.$, treatment $=0.950$; hemisphere $=0.153$; treatment $*$ hemisphere $=0.017 ; p>0.343$; Fig. 4 \& Table 3; see Fig. 2 for experimental timeline). Therefore, contrary to our original prediction, unilateral inhibition of estrogen synthesis in NCM did not impair tutor song memorization and eventual imitation.

Manual song similarity quantification. Whole motif similarity measurements via SAP is the conventional method to objectively analyze tutor similarity for zebra finches ${ }^{62}$. Inspection of spectrograms suggested that SAP similarity measurements were not capturing the full extent of tutor song similarity (Supp. Fig. 2B: high \% SAP song similarity for Cannula subject [surgery-control animal], but visually and acoustically dissimilar; opposite issue with $a C S F$ subject). To address this, we employed visual song similarity measures in the spirit of early songbird bioacoustic research studies that relied solely on visual spectrographic assessment ${ }^{63-65}$. In accordance with this match between SAP and when visual scoring methods, there were no significant effects for visually-scored song similarity (average percent copied) by cannulated hemisphere $\left(F_{(1,16)}=0.227, p=0.640\right)$, treatment $\left(F_{(1,16)}=0.561, p=0.465\right)$, nor an interaction between either factor $\left(F_{(1,16)}=0.074, p=0.789\right.$; Supp. Fig. 2C). Therefore, irrespective of bioacoustic assessment, unilateral blockade of neuroestrogen production in the auditory forebrain during and immediately after song learning did not impair auditory memorization of the tutor song. 

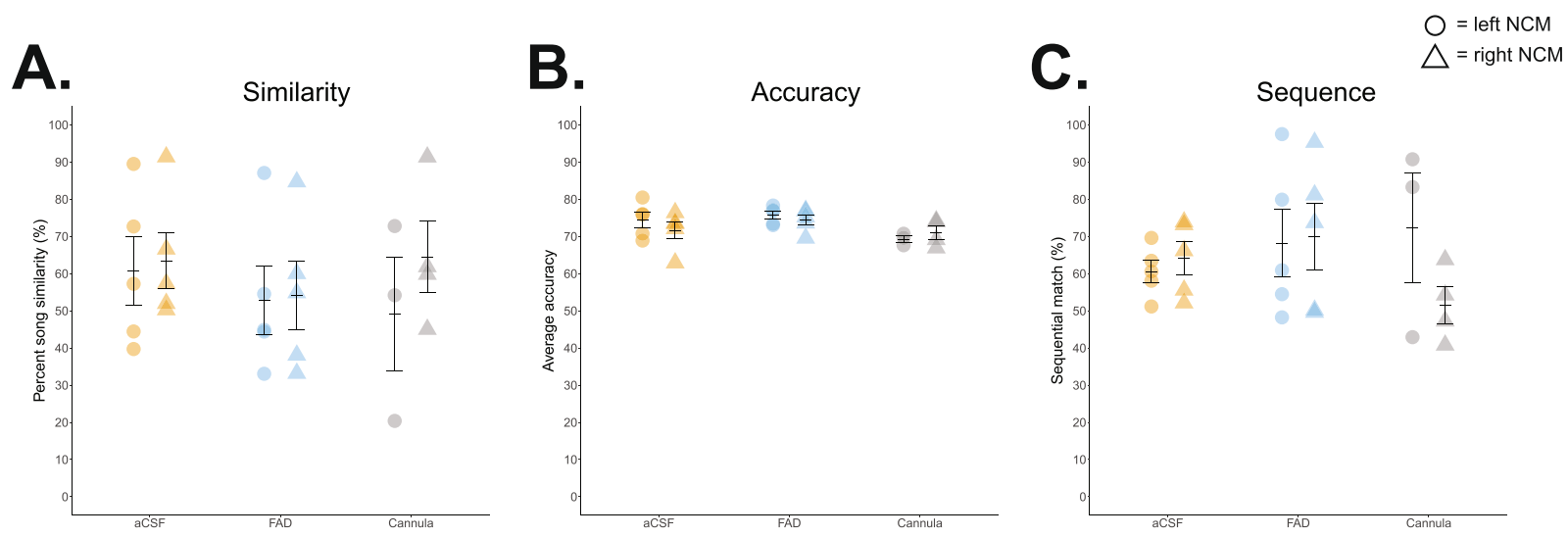

Figure 4. Song copying is unaffected when neuroestrogen production is inhibited via in vivo microdialysis. $130 \mathrm{dph}$ (A) song similarity, (B) accuracy, and (C), sequence similarity, respectively, are all comparable across aCSF- and FAD-treated birds. Cannula 'surgery controls' are graphed for visual comparison. Orange $=\mathrm{aCSF}$; blue $=\mathrm{FAD}$; grey $=$ cannula; circle $=$ left $\mathrm{NCM}$; triangle $=$ right $\mathrm{NCM}$.
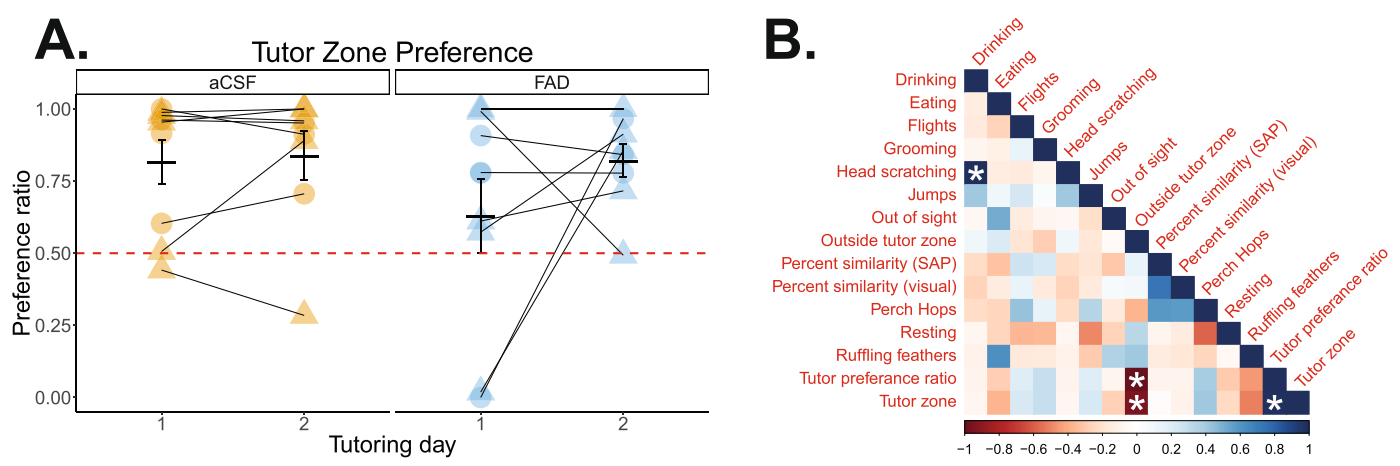

Figure 5. Juvenile male songbirds are similarly attentive to the tutor during microdialysis. (A) The time a bird spent near a live adult male tutor during in vivo microdialysis is similar across treatments, targeted hemispheres, and tutoring day. Behavior presented is from the first 10 minutes of song playback alongside live male presentation (see Methods). Orange $=\mathrm{aCSF}$; blue $=\mathrm{FAD}$; circle $=$ left $\mathrm{NCM}$; triangle $=$ right $\mathrm{NCM}$. (B) Correlogram of tutoring behavior and song similarity measurements reveal significant correlations (more time spent near the tutor negatively associated with time spent away from the tutor; tutor zone time positively correlated with tutor preference ratio), and novel findings (positive correlation of head scratching and drinking); $p<0.0005$ (adjusted $\alpha$; Bonferroni correction). Behavior data presented is from the first 10 minutes of tutor playback across days 1 and 2 of tutoring.

Tutoring behavior. Attention plays a critical role for vocal learning early in development ${ }^{66}$. Since estrogens can modulate attention in rodents ${ }^{67}$, we explored whether FAD treatment impaired measures of attention during tutoring sessions in a subset of subjects (FAD $n=9$; aCSF $n=9$ ). Overall, we found no effect of treatment on the amount of time pupils spent near the tutor ('tutor zone'; a proxy for tutor attention) on either tutoring day ( $F_{(1,}$ ${ }_{14)} ; p>0.190$ for main effects and interaction; Fig. 5A). We also explored whether the other behaviors we scored might also be predictive of future tutor song similarity. We generated a correlogram that included all tutor session behaviors, as well as song similarity measurements (both visual and SAP derived). Overall, there were few significant correlations of interest pertaining to song similarity and behavior that emerged (Fig. 5B).

Another possibility is that FAD treatment may impair locomotion. We explored whether two common motor behaviors (jumping and perch hopping), as well as time spent resting/sleeping were affected by pharmacological exposure. Overall, neither treatment nor tutoring day affected jumping or perch hops $\left(F_{(1,14)} ; p>0.158\right.$ for main effects and interaction; Supp. Fig. 3A,B); however, birds spent more time resting irrespective of treatment on the first day of tutoring, suggesting that the novelty of an adult male increases after the first session $\left(\mathrm{F}_{(1,14)}=7.938\right.$, $p=0.0137$; all other analyses $p>0.808$; Supp. Fig. $3 \mathrm{C}$ ). These results suggest that, as with song similarity, behavior during a social learning session is unaffected by unilateral blockade of estrogen synthesis in NCM.

Song changes after exposure to adult male conspecifics. We noticed highly aberrant song types in several formerly microdialyzed subjects independent of treatment at $131 \mathrm{dph}\left(X^{2}(N=23)=1.189, p=0.552\right)$, which is well beyond the putative 'closing' of the critical period for tutor song memorization and song should be highly stable (Supp. Fig. 4). Aberrant songs were always highly variable (i.e. not crystallized/stereotyped) at $130 \mathrm{dph}$ and eventually reverted to higher stereotypy after being exposed to other adult male birds, and typically involved 
A.
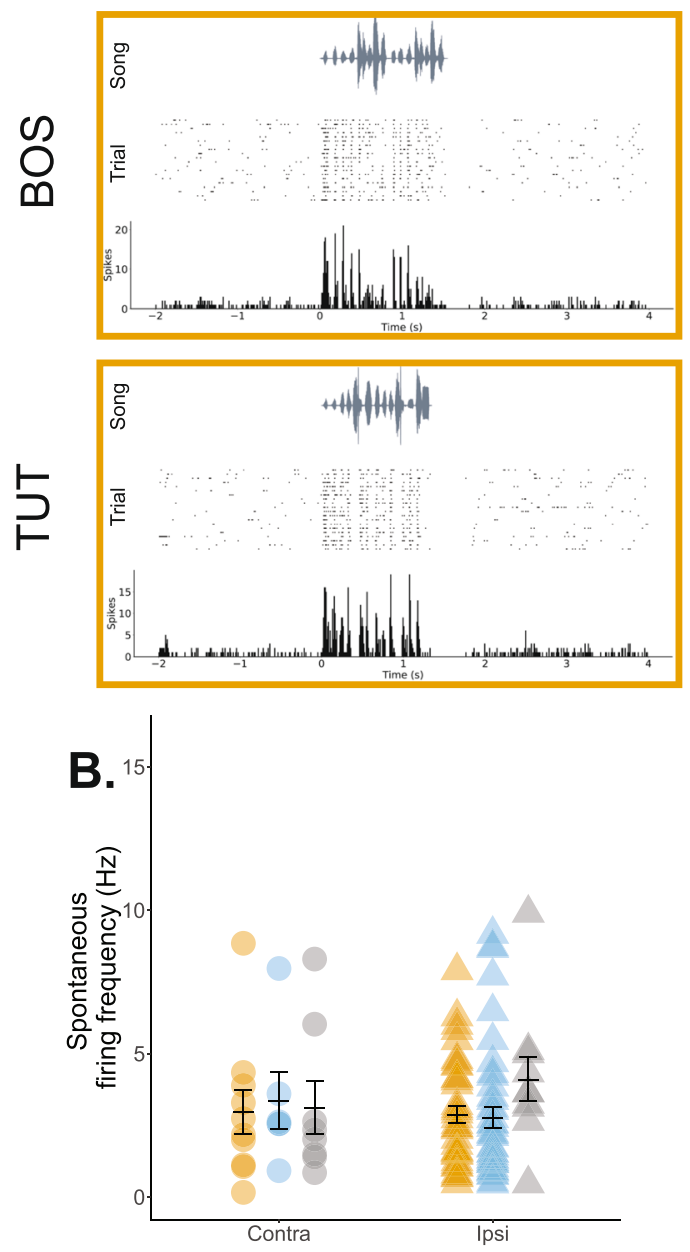

D.

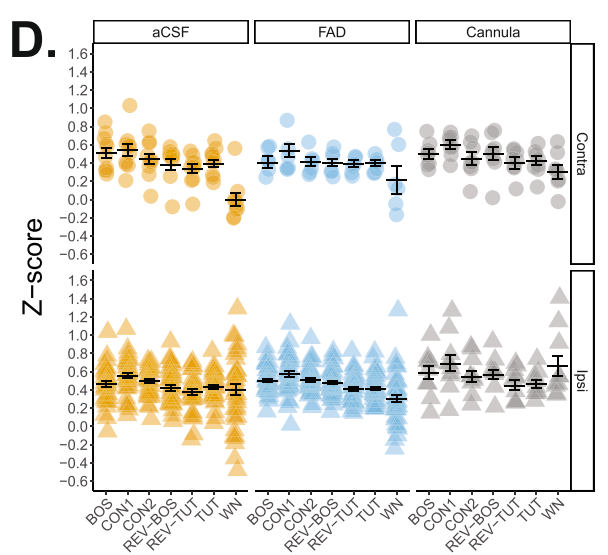

FAD
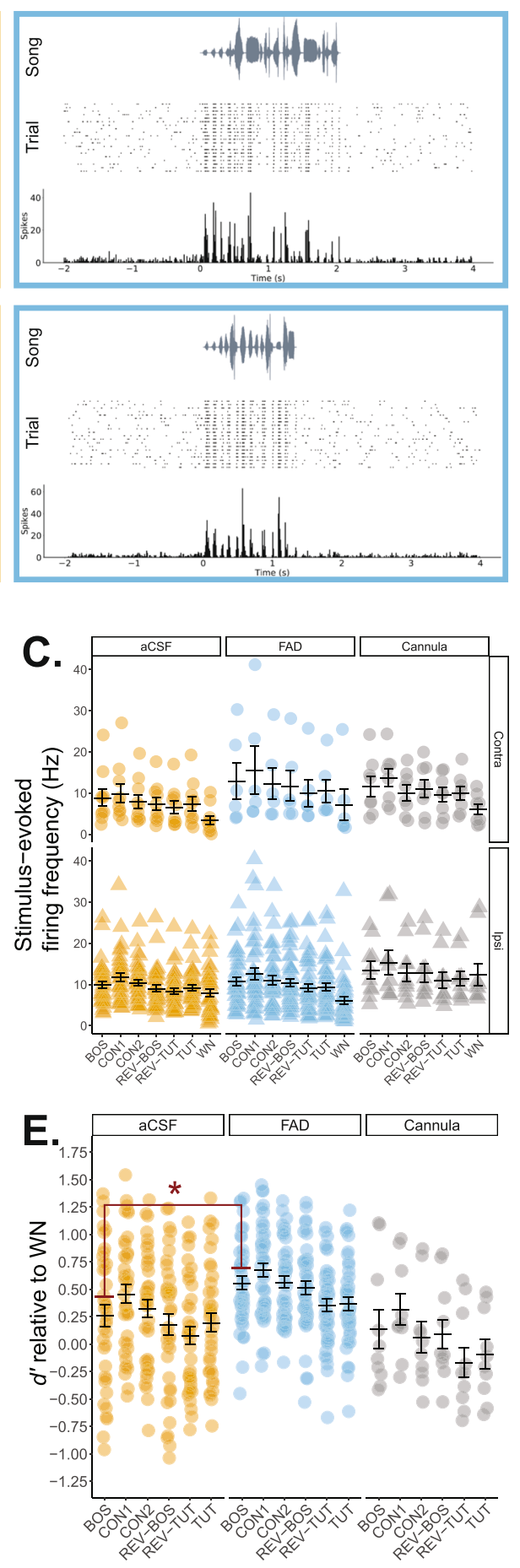

Figure 6. Single-unit recordings in NCM reveal modest differences in auditory responses in adulthood. (A) Representative NCM single-unit recordings from an aCSF and FAD in response to presentations of birds' own song (BOS) and tutor song. Each recording includes a song spectrogram (Top), and raster plot (Middle) with corresponding peri-stimulus time histogram in $10 \mathrm{~ms}$ bins (Bottom) across a 6 second period. The same unit is presented for each treatment across the two stimuli. (B) Spontaneous firing rates were unaffected by developmental microdialysis treatment. Orange $=\mathrm{aCSF}$; blue $=\mathrm{FAD}$; grey $=$ cannula; circle $=$ contralateral hemisphere (relative to microdialysis site); triangle = ipsilateral hemisphere (relative to microdialysis site). (C) Stimulus-evoked firing rates were significantly lower for WN and overall higher for CON1. A recording hemisphere $\times$ treatment interaction was significant; however, post hoc analyses limited to CON1 found no statistical differences for either treatment. (D) Analysis of normalized auditory response (z-score) yielded a significant stimulus $\times$ recording hemisphere effect: contralateral NCM responded less to WN compared to all other stimuli, whereas forward conspecific stimuli elicited higher responses in the ipsilateral NCM, irrespective 
of treatment. (E) Ipsilateral d'values relative to WN. BOS selectivity was higher in FAD songbirds in the ipsilateral hemisphere. $\mathrm{BOS}=$ birds' own song; CON1; CON2 = conspecific song; REV-BOS = reverse bird's own song; REV-TUT $=$ reverse tutor song; $\mathrm{TUT}=$ tutor song. ${ }^{*} p<0.05$.

dropping and/or adding new syllables (6/8 subjects added, dropped, or modified syllables). These results further emphasizes that experience gates song crystallization, which has been described in other studies on lab-reared tutored and isolate zebra finches ${ }^{65,68-70}$. It is also possible that the process of microdialysis itself, including attendant neurochemical, neuroinflammatory, or other reactive events were partially responsible for the developmental delay in song sensitive period closure.

Neuroestrogen suppression in development leads to enhanced neural representations of birds' own song and tutor song in HVC of adults. In a subset of formerly microdialyzed birds ( 21 out of 28 birds), we obtained neural recordings from two brain regions associated with song learning and tutor memory representation: NCM and HVC. Recordings were obtained from both the contralateral and ipsilateral hemisphere relative to the site of microdialysis cannulation (i.e. contralateral= recording from non-dialyzed hemisphere; ipsilateral $=$ recording from dialyzed hemisphere).

NCM. We first explored whether treatment impacted NCM firing properties. Spontaneous firing rates were unaffected by recording hemisphere, treatment, and there was no interaction between the two factors $\left(F_{(1}\right.$ $\left.{ }_{93}\right)=0.238,0.003$, and 0.779 , respectively; $p>0.60$; Fig. $\left.6 \mathrm{~B}\right)$. Contrary to spontaneous firing, stimulus-evoked firing was significantly affected by a recording hemisphere $\mathrm{x}$ treatment interaction $\left(F_{(1,651)}=7.938, p=0.005\right)$ as well as there being a main effect for treatment $\left(F_{(1,6)}=4.334, p=0.038\right)$ and stimulus $\left(F_{(6,651)}=7.670, p<0.001\right)$. Follow-up analyses revealed that the stimulus effect was driven mainly by an overall lower response to WN (WN < BOS, CON1, CON2, and REV-BOS), and a higher response evoked by CON1 (CON1 > REV-TUT; Tukey's HSD, $p<0.02$ for all stimulus comparison; all post-hocs were corrected for multiple comparisons here and throughout; Fig. 6C). To avoid pseudo-replication ${ }^{71}$, and because of the main effect of stimulus, we opted to perform follow-up analyses on just CON1 data for NCM. Follow-up analyses did not yield any significant differences between recording hemispheres for stimulus-evoked firing in FAD-treated $\left(F_{(1,46)}=0.513, p=0.478\right)$ nor aCSF-treated subjects $\left(F_{(1,47)}=0.734, p=0.396\right)$.

While raw firing rate data are informative, it is also useful to consider normalized auditory response rates ( $z$-score) which accounts for recording site variability in spontaneous and stimulus-evoked activity e.g. ${ }^{40}$. Analyses revealed a significant main effect of stimulus $\left(F_{(6,651)}=17.643, p<0.001\right)$ and recording hemisphere $\left(F_{(1,651)}=12.935, p<0.001\right)$, as well as a significant interaction between stimulus and recording hemisphere $\left(F_{(6}\right.$, ${ }_{651)}=3.051, p=0.006$; Fig. 6D). In contralateral NCM, WN elicited a significantly lower z-score compared to all other stimuli ( $p<0.001$ for all stimulus comparisons). In contrast, $z$-scores were typically higher for non-reversed conspecific stimuli in the ipsilateral hemisphere regardless of treatment (CON1 > REV-BOS, REV-TUT, TUT, and $\mathrm{WN}$; BOS $>$ REV-TUT and WN; CON2 $>$ REV-TUT and WN; $p<0.05$ for all stimulus comparisons). Overall, the results in NCM suggest that irrespective of treatment, forward, conspecific stimuli (i.e. CON1, CON2, and BOS) reliably evoke the highest normalized auditory responses in the cannulated hemisphere.

Our initial impetus in recording from microdialyzed subjects was to test whether representations of learned stimuli (i.e. BOS and TUT) were different based on treatment early in development. To address this question, we calculated d prime ( $\boldsymbol{d}$ '; see Methods) relative to WN to determine stimulus selectivity, as described in previous studies $^{7,72,73}$. We limited our analyses to TUT and BOS as these were the learning-related auditory stimuli of interest that may have been impacted by treatment. Because of our earlier findings for auditory response profiles, we compared $d$ ' scores separately by recording hemisphere. Treatment did not impact overall TUT selectivity for either contralateral $\left(F_{(1,14)}=2.222, p=0.158\right)$ or ipsilateral $\left(F_{(1,79)}=2.861, p=0.095\right)$ recording sites in NCM. However, FAD subjects demonstrated significantly stronger BOS selectivity in the ipsilateral cannulated $\left(F_{(1}\right.$, ${ }_{79)}=6.371, p=0.014$; Fig. $\left.6 \mathrm{E}\right)$, but not contralateral hemisphere $\left(F_{(1,14)}=3.93, p=0.067\right.$; Supp. Fig. 5A $)$. Taken together, unilateral E2 suppression in NCM during development enhances BOS representation in NCM relative to control birds.

HVC. The sensorimotor nucleus HVC contains a population of tutor-song-selective cells ${ }^{73-76}$ and receives E2-sensitive, indirect projections from NCM in part via the nucleus interfacialis of the nidopallium Nif ${ }^{39,77}$. To determine whether suppressing E2 synthesis in development affected downstream representations of either BOS or tutor song, we also recorded from HVC. As microdialysis treatment was restricted to NCM and could not reach HVC, nor Nif, any change in HVC response properties would be due to the indirect influence of modulatory events in NCM that propagate into HVC. Baseline firing rates were similar across treatments, recording hemisphere, and no interaction between the two factors were found $\left(F_{(1,47)}, p>0.132\right.$; Fig. 7B). For stimulus-evoked firing, there was a main effect of stimulus $\left(F_{(6,329)}=5.83, p<0.001\right)$ and recording hemisphere (ipsilateral $>$ contralateral; $F_{(1,329)}=10.661, p=0.001$; Fig. $\left.7 \mathrm{C}\right)$. All other effects and interactions were non-significant $(p>0.131)$. Follow-up analyses revealed that, as expected, BOS elicited a significantly higher evoked firing response compared to all stimuli except TUT (BOS $>$ CON1, CON2, REV-BOS, REV-TUT, and WN; $p<0.05$ ); no other stimulus comparisons were significantly different.

As with NCM, we also analyzed normalized auditory response in HVC. There was a significant effect of stimulus $\left(F_{(6,329)}=10.384, p<0.001\right)$, treatment $\left(F_{(1,329)}=11.297, p<0.001\right)$, as well as a significant interaction between recording hemisphere and treatment $\left(F_{(1,329)}=25.745, p<0.001\right.$; Fig. 7D). All other main effects and interactions 

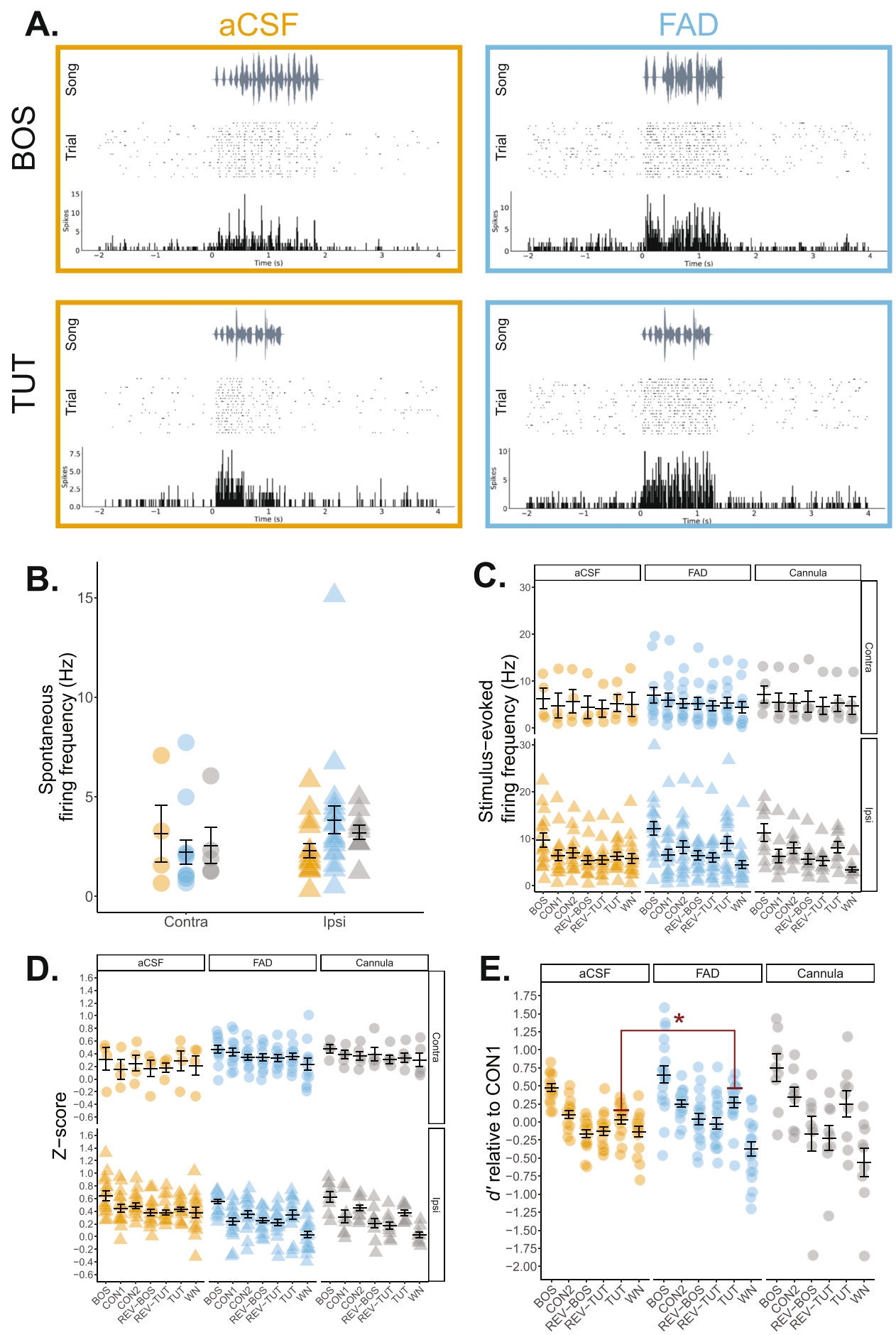

Figure 7. Tutor song selectivity is elevated in single HVC neurons of formerly estrogen-suppressed adult songbirds. (A) Representative HVC single-unit recordings from an aCSF and FAD in response to presentations of birds' own song (BOS) and tutor song. Each recording includes a song spectrogram (Top), and raster plot (Middle) with corresponding peri-stimulus time histogram in $10 \mathrm{~ms}$ bins (Bottom) across a 6 second period. The same unit is presented for each treatment across the two stimuli. (B) Spontaneous firing rates were similar across treatments. Orange $=\mathrm{aCSF} ;$ blue $=\mathrm{FAD}$; grey $=$ cannula; circle $=$ contralateral hemisphere (relative to microdialysis site); triangle $=$ ipsilateral hemisphere (relative to microdialysis site). (C) Stimulus-evoked firing rates were significantly higher for BOS compared to all other stimuli except for TUT. Further, ipsilateral HVC displayed higher overall stimulus-evoked firing rates compared to contralateral HVC, independent of treatment. (D) Analysis of normalized auditory response (z-score) yielded similar results as with firing rate; namely, a significantly higher response to BOS over all other stimuli independent of treatment, as well as a significantly suppressed response to WN compared to CON2 and TUT. (E) Contralateral d' values relative to CON1. TUT selectivity is significantly higher in FAD subjects solely in the contralateral hemisphere. BOS = bird's own song; CON1; CON2= conspecific song; REV-BOS = reverse bird's own song; REV-TUT $=$ reverse tutor song; $\mathrm{TUT}=$ tutor song. $* p<0.05$. 
A.

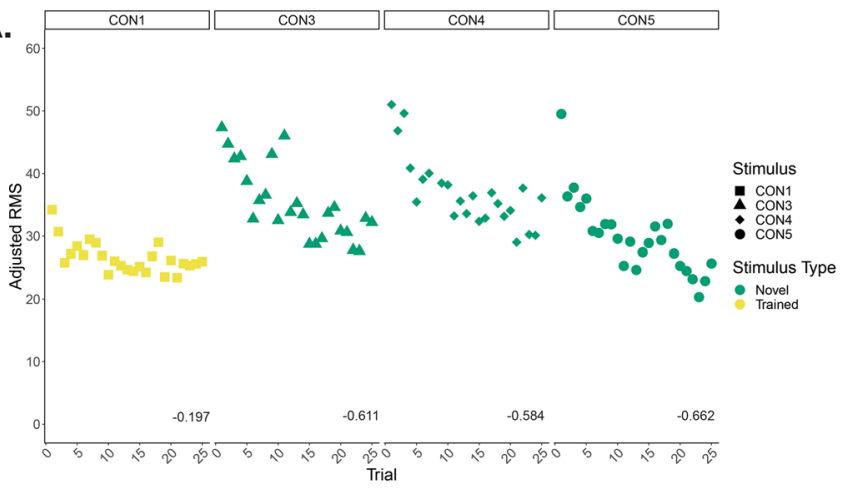

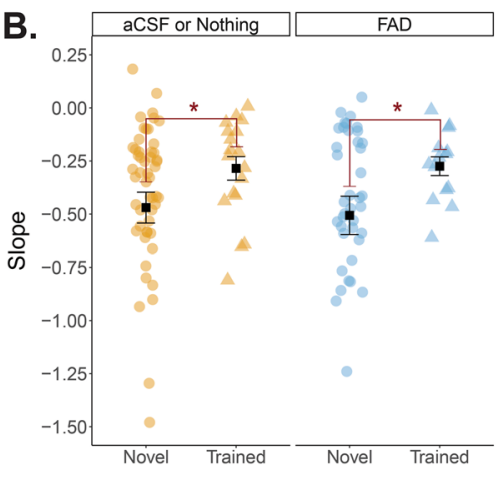

Figure 8. Neural adaptation to learned song is reduced in adult NCM independent of post-training E2 synthesis inhibition. (A) An exemplar multiunit response in the NCM of an untreated hemisphere. Adjusted RMS declines at a faster rate (steeper slope) for novel song (CON3, CON4, and CON5) compared to a shallower slope (slower adaptation) for the recently exposed song (CON1). Slopes for each stimulus is shown at the bottom of each panel. (B) Average slope per stimulus in aCSF or non-treated hemispheres compared to FADtreated hemispheres in NCM; slope derived from multi-unit RMS. Orange $=\mathrm{aCSF}$ or no treatment; blue $=\mathrm{FAD}$; circles $=$ novel stimuli (three unique $\mathrm{CON}$ per bird); triangles $=$ trained stimulus (a single unique $\mathrm{CON}$ ). The $\mathrm{y}$-axis has been compressed for clarity and five slope data points were omitted $(-3.37,-2.72,-2.56,-1.58$, 0.51). $*$ = significant main effect of stimulus type (novel vs. trained); $p<0.05$. $\mathrm{CON}=$ conspecific song.

were non-significant $(p>0.176)$. As expected, BOS elicited a significantly higher response than did all other stimuli (BOS $>$ CON1, CON2, REV-BOS, REV-TUT, TUT, and WN; $p<0.016)$. Conversely, HVC was less responsive to WN compared to select forward conspecific stimuli (WN $<$ CON2 and TUT; $p<0.009$ ). Based on the enhanced response to BOS for both z-score and stimulus-evoked firing, we opted to focus our follow-up tests on BOS. No significant differences were found for treatment for either the contralateral $\left(F_{(1,14)}=1.097, p=0.313\right)$ or the ipsilateral $\left(F_{(1,33)}=1.223, p=0.277\right)$ hemisphere.

For selectivity analyses, we focused solely on BOS and TUT relative to CON1 and tested whether TUT and BOS were differently represented between treatments. As there was a significant effect of stimulus and recording hemisphere, we analyzed the effect of treatment on TUT and BOS selectivity separately by hemisphere. BOS selectivity was statistically similar across treatments across both the ipsilateral $\left(F_{(1,33)}=1.691, p=0.202\right.$; Fig. 7E), and contralateral hemisphere $\left(F_{(1,14)}=0.804, p=0.385\right.$; Supp. Fig. $\left.5 B\right)$. In contrast, HVC units were more selective for TUT in the ipsilateral hemisphere of FAD subjects $\left(F_{(1,33)}=5.82, p=0.022\right.$; Fig. $\left.7 \mathrm{E}\right)$, but not contralateral hemisphere $\left(F_{(1,14)}=3.45, p=0.084\right.$; Supp. Fig. $\left.5 \mathrm{~B}\right)$. Taken together, unilateral E2 synthesis inhibition in NCM enhanced the neural selectivity for tutor song in HVC, without causing changes in eventual tutor song imitation. It is possible, therefore, that the alteration of neuroestrogen synthesis in NCM shifts the tutor song representation in the auditory/song system (including HVC), but that this is compensated for during the course of subsequent development and does not markedly affect tutor song similarity.

Adult songbirds are unaffected by post-training inhibition of estrogen synthesis in NCM. As with juvenile songbirds, E2 is also acutely synthesized in the NCM of adult songbirds ${ }^{78,79}$. Therefore, we also tested whether neuroestrogen production is involved in consolidating recent auditory experience in adult male zebra finches using a well-established auditory adaptation paradigm (see Methods). A conventional metric to auditory memory in adults is to compare the decreasing rate at which cells fires to different songs (slope, or a related index based on slope $)^{42}$. If a song is "recognized" (familiar), then adaptation rates should be shallower (lower), whereas a unfamiliar ("novel") song should have a steeper (higher) slope. Overall, adaptation rates (slope) were significantly shallower (lower) for familiar vs. novel stimuli (familiar $=-0.28 \pm 0.4$, novel $=-0.49 \pm 0.06 ; F_{(1}$, $\left.{ }_{122)}=4.150, p=0.044\right)$, independent of treatment $\left(F_{(2,122)}=1.182, p=0.310\right)$ or an interaction between treatment and stimulus type $\left(F_{(2,122)}=0.349, p=0.706\right.$; Fig. 8$)$. Thus, unilateral estrogen synthesis in NCM immediately post-training did not adversely impact memory consolidation across development and in adulthood.

\section{Discussion}

Our collective findings indicate that while aromatase is present in developing auditory cortex, systemic and unilateral attenuation of neuroestrogen production does not impair tutor song memorization. However, neuroestrogen blockade leads to suppressed song production during development and enhanced neural representations of tutor song in a downstream sensorimotor nucleus when measured in adulthood. Therefore, contrary to E2's presence facilitating hippocampal-dependent adult spatial memory in a variety of species, downregulation of neuroestrogens may be permissive for auditory memorization, and its synthesis may be primarily important for exerting an effect on communication production and representation throughout the lifespan in songbirds. Taken together, this study is the first, to our knowledge, that tests the involvement of estrogen synthesis in consolidating an ethologically-relevant sensory memory within the developing auditory forebrain. Therefore, this study extends our knowledge of the role, region, and age in which estrogen is involved in learning.

We observed a decline in NCM cell density in sensorimotor-aged birds compared to sensory-aged subjects. Only one prior study, to our knowledge, has assessed the cell density of NCM across development and found no 
regional nor age differences in sensory-aged $(20$ and $30 \mathrm{dph})$ and adult male zebra finches ${ }^{80}$. It is unclear why our results diverge from those of Stripling, et al. ${ }^{80}$, other than the resolution in the current study of sensorimotor vs sensory stages. Our findings suggest a form of age-dependent network pruning that is consistent with heightened auditory responses in NCM in sensory- vs. sensorimotor-aged male songbirds ${ }^{40}$. Alternatively, the volume of NCM may expand with age, leading to decreased neuronal density. To our knowledge, the volume of NCM across development has not been well characterized, and these ideas remain to be tested.

The density of cells in dorsal NCM was higher compared to ventral NCM, in contrast to previous observations $^{80}$. This effect was independent of age, suggesting an anatomical distinction in developing NCM that may persist in adulthood (M. Macedo-Lima \& L. Remage-Healey, unpublished observations). Numerous studies have described dorsal/ventral differences in response to auditory stimuli in NCM, but there does not appear to be a consensus regional effect. For example, immediate-early gene (IEG) auditory responses (i.e. ZENK induction in NCM in response to auditory playbacks) yield varying results depending on species: no differences between NCM subregions are reported in in adult male European starlings ${ }^{81}$ or adult male budgerigars ${ }^{82}$, whereas higher dNCM ZENK compared to vNCM has been reported in both adult female white-crowned sparrows ${ }^{83}$ and both sexes of adult black-capped chickadees ${ }^{84}$, but see ${ }^{85}$. In contrast, extracellular recordings in the NCM of adult starlings find stronger experience-dependent changes in firing rates in ventral vs dorsal $\mathrm{NCM}^{86}$, which were suggested to be attributed to a noted enhanced thalamic input from Field L to ventral NCM (Vates et al. 1996). Therefore, while subregions of NCM differ in cell density, the functional significance of this density difference across development is unclear, but are suggestive of regional differences in auditory responsiveness ${ }^{87}$.

In addition to quantifying NCM cell density, we found that levels of both aromatase and parvalbumin were unchanged across development. While aromatase expression has been previously assessed across development and in adults, we found that subregions within NCM of sensory- and sensorimotor-aged males possess a similar numerical capacity for estrogen synthesis. As aromatase is similarly expressed in NCM across development, changes in precursor androgens may explain previously observed age-dependent differences in baseline E2 in NCM across the critical period (Chao, et al., 2014), specifically in parallel with the maturation of the testes. Further, our findings with parvalbumin are in-line with recent findings that find that PV cell density is largely unchanged across development in the NCM of in male and female zebra finches, as well as other auditory forebrain nuclei ${ }^{88}$. Therefore, PV-dependent inhibitory tone and estrogen production remain relatively unchanged across development, suggesting important roles throughout the juvenile period in males; however, further work is necessary to determine if their presence is functionally significant in ontogeny.

Our experiments with systemic FAD treatment suggest that E2 facilitates song production in juvenile songbirds. It is well established that singing is regulated by classic (genomic) steroid hormone action, such as E2, in adult songbirds. In adult male zebra finches, long-term aromatase inhibition leads to suppressed courtship displays, including song production ${ }^{89}$. More recent studies have found that neuroestrogen production also appears to acutely facilitate song production in adult zebra finches ${ }^{59,90}$. Our data expand on this understanding that acute suppression of E2 production constrains singing to now include developing male songbirds. The neural locus of this effect of E2-withdrawal on song production is unknown, but likely to include social behavior network nuclei such as the aromatase-rich nucleus taenia ${ }^{50,91}$. Androgens, namely testosterone, have classically been thought to be the critical hormone for the onset of motor production in developing songbirds ${ }^{92}$. For example, plastic song emerges alongside the rise of testosterone in juvenile swamp sparrows ${ }^{45}$. However, it has also been noted that circulating estrogens also coincides with the onset of subsong ${ }^{45}$. Thus, our data suggest that E2, and the conversion of precursor androgens to E2 within specific brain areas, may play a more significant role in song production in development than previously thought.

Overall, systemic aromatase inhibition yielded minimal effects on eventual tutor song similarity. These results are novel given the relatively limited number of studies that have directly tested the role of hormones in song learning in male songbirds. Androgens are associated with the crystallization of plastic song ${ }^{92-94}$ and neural circuit development ${ }^{95}$. In contrast, circulating estrogen levels are thought to promote plasticity due to their coincident rise in age-limited song learning in birds during the auditory memorization ("sensory") phase of development ${ }^{43-46}$, but see $^{96}$. While our sample size is limited, the data suggest that circulating estrogen synthesis is not required for tutor song memorization during development.

One important caveat for the systemic FAD experiment here is that our pharmacological treatment may have missed a putative 'critical' post-training consolidation period (e.g., within the first $\sim 30$ mins following tutoring). E2 is important for auditory processing in adult and juvenile songbirds; thus, we did not want to interfere with online auditory processing of the tutor song during a tutoring session/playback. Instead, we intentionally administered FAD immediately after the offset of tutoring to specifically target the post-training memory consolidation period as in studies on hippocampal E2 and memorization ${ }^{97}$. Acute administrations of an aromatase inhibitor via peripheral injection in birds markedly reduces forebrain E2 levels within 6 hours $^{58}$, and aromatase activity within $\sim 30$ minutes $^{98}$, including zebra finch $\mathrm{NCM}^{59}$. Thus, if systemic FAD takes 30 minutes to suppress E2 synthesis after administration, and the putative auditory memory consolidation period is potentially "over" 30 minutes following the cessation of a training experience, our treatment (FAD) may have not aligned with the target phase (post-training consolidation phase). That is, orally administered FAD may not sufficiently suppressed telencephalic E2 levels in pupils during the brief period of tutor song memory consolidation following a tutoring session. Moreover, our study design also leaves open the possibility that local estradiol levels fluctuate during 'off days' in systemic birds when tutoring nor treatment occurred.

In agreement with our systemic results, targeted unilateral suppression of neuroestrogen synthesis in NCM failed to prevent birds from eventually successfully imitating their tutor's song. Tutoring leads to an initial drop in acute E2 levels within NCM, followed by a rapid increase immediately after a tutoring session in juvenile songbirds ${ }^{49}$. In our paradigm, FAD was presented at the onset of tutoring and for a one-hour period immediately 
following the tutor session, without any detectable differences in eventual song similarity. Therefore, unilateral E2 synthesis in NCM does not appear to be required for auditory memory consolidation.

Additionally, juvenile songbird behavior is seemingly unaffected by unilateral estrogen manipulations in the auditory forebrain. Birds spent comparable amounts of time near by the live tutor and were similarly active during tutoring sessions. These results add to a small but growing understanding of tutor and pupil behavior during song learning. To our knowledge, these results are novel given the limited studies that explicitly quantify pupil behavior during tutoring (lab-reared, or otherwise $)^{6,66,99-101}$. Juveniles are thought to preferentially learn from, and as an extension, imitate, more aggressive males who are mated or feed them early in development ${ }^{61}$. While it is largely unknown how pupil behavior during tutoring affects song learning, one key behavior appears to be pupil 'attention' during tutoring ${ }^{6,66}$. Our study found that unilateral E2 synthesis did not impact attention, as quantified by time spent near the tutor, thereby not interfering with song learning and imitation. However, an important caveat to our findings is that due to animals being tethered, we did not quantify pupil's head position (i.e. facing tutor or not), which Ahmadiantehrana \& London (2017) found to be a more reliable predictor for song learning.

Suppressing E2 in NCM during development led to enhanced adult neural representations of the tutor's song in HVC. HVC is a sensorimotor nucleus that dually represents both autogenous and tutor song in developing $^{75,102,103}$ and adult ${ }^{73,104}$ songbirds, and is necessary for song learning ${ }^{105}$. One possibility is that if neuroestrogen blockade reduces singing in microdialyzed birds as in our systemic experiments, there may be a 'catch-up' period that leads to enhanced salience, coding, or replay ${ }^{106}$ of the social model's song (tutor) once E2 synthesis inhibition is 'released' in NCM. Thus, our findings are consistent with other recent findings in swamp sparrows, in which HVC tutor- and BOS-selectivity is independent of vocal imitation accuracy in adulthood ${ }^{73}$. This interpretation is also consistent with the role of NCM E2 in regulating local (NCM) neural responses to songs, as well as regulating downstream response selectivity for BOS in HVC, as was demonstrated using similar methods in adult male zebra finches ${ }^{39}$. Clearly, therefore, the estrogenic neuromodulatory events in NCM can have impacts on the sensorimotor song pathway in juveniles and adults, but the implications of this for storage and recall of auditory memories are still unclear. Moreover, the general processing stream of information as it progresses from the auditory NCM and $\mathrm{HVC}$ are becoming clearer ${ }^{107}$, but a direct anatomical basis for this physiological connection remains to be elucidated.

Interestingly, FAD treatments enhanced BOS selectivity in NCM compared to control birds. Auditory forebrain neurons (including NCM) are typically selective for conspecific vocalizations over synthetic noises (e.g. tones) ${ }^{80,108}$, and contain a subpopulation of BOS-selective cells ${ }^{7,109-111}$. In particular, NCM contains experience-dependent tutor song and dual tutor song/BOS selective neurons during development ${ }^{7}$. Auditory responses in NCM are rapidly modulated by estrogens in adult ${ }^{38,39}$ and developing zebra finches ${ }^{40}$. Therefore, in agreement with our findings in HVC with tutor song, acute manipulations of E2 in NCM during development appear to be important for changing representations of birds' own song as well.

It is worth noting that our treatments were presented unilaterally, and there is thus a strong likelihood that contralateral NCM can compensate for depressed E2 production in our study, leading to robust tutor song memory and proper song imitation in adulthood. While NCM appears to have lateralized function both natively ${ }^{112}$, and with regard to the acute actions of $\mathrm{E}^{38,113}$, there is scant evidence for lateralized expression of aromatase $\mathrm{s}^{50,55}$. Relatedly, there is the additional possibility that either acute (microdialysis) or chronic (systemic) administrations may lead to homeostatic increases in aromatase production and/or activity e.g. ${ }^{50}$, or upregulation of E2 from other sources (e.g. gonadal; adrenal). For example, estrogen-suppressed adult zebra finches have increased aromatase protein levels in the hippocampus, but not $\mathrm{NCM}^{50}$. Lastly, it is possible that cannulation-induced injuries across control and FAD treated subjects obscured any potential differences in song learning outcomes. That is, since guide cannulae dissociated on their own, brain injury from the cannula may lead to similarly poor song learning outcomes as with FAD treatment (just as cannulation itself may have led to differences in neuronal auditory-response rates as in Fig. 6C). However, this possibility is unlikely to explain our findings as both microdialysis and systemically treated birds yielded comparable tutor song similarity in adulthood. Further, other studies wherein developing songbirds are implanted with bilateral cannulae produce normal song in adulthood ${ }^{5,6}$.

Our study also replicates and extends prior observations that experience with social partners, in addition to age, can regulate the closure of the critical period in songbirds. Importantly, the lack of song crystallization by 130 dph was independent of treatment, further emphasizing that unilateral estrogen synthesis in NCM does not participate in modulating critical period plasticity in contrast to androgens which prematurely crystallize song and related neural circuits (reviewed above). Others have also noted abnormal song in adulthood in lab-tutored songbirds $^{61,65,68-70,114,115}$, and found similar changes such as dropped syllables, reduced syllable lengths, and increased stereotypy once abnormal singing birds were exposed to other adult males. Our work highlights the important limitation of controlled lab tutoring paradigms, namely that it is both quality and quantity of experience that dictate the closure of critical period song plasticity.

Our results in adult animals build on a well-established paradigm in which recent auditory experience is encoded in adult and developing NCM ${ }^{108,116-119}$. Repeated exposures of a single conspecific song leads to neural 'recognition' up to 48 hours later ${ }^{116}$, which is impaired when global estrogen production is dampened ${ }^{42}$. As in prior reports, we observed auditory recognition memory in the NCM of adults; however, post-training unilateral inhibition of E2 synthesis did not impair this recognition memory. Our findings suggest that post-training unilateral E2 availability may not be required for consolidation within the NCM of songbirds. This is in contrast to evidence suggesting E2's importance in consolidating other sensory memories (e.g. chemosensory in rodent olfactory bulb ${ }^{120}$ ) and spatial memory consolidation in the hippocampus of songbirds and rodents ${ }^{26,97}$. In rodents, E2 is rapidly upregulated in dorsal hippocampus immediately following an object recognition training session ${ }^{28}$. In contrast, repeated song exposure in adult male and female zebra finches leads to an immediate increase in estrogen levels that tapers off following cessation of song playback or social exposure ${ }^{78,79}$. Since adult NCM E2 
levels after song exposure are similar to baseline, E2 may be required for both auditory processing and consolidation only while birds are listening to song. Therefore, blocking E2 after song exposure may leave recent auditory memories unimpaired in adults because it "misses" the window of E2's influence.

Here, we demonstrate that estrogens exert a complex role in the auditory cortex of developing male songbirds. Our findings show the capacity to synthesize neuroestrogens remains high throughout development alongside substantial age- and subregion-dependent changes in NCM cell density. Systemic estrogen synthesis blockade led initially to suppressed singing behavior in juveniles following tutoring. Further, while song memorization was unimpaired by acute inhibition of E2 production following training in developing and adult songbirds, early life E2 manipulations in auditory forebrain lead to altered neural selectivity of autogenous and tutor song in NCM and downstream HVC in adulthood, respectively. Taken together, this study expands our understanding of the role of brain-derived estrogens in learning and memory. In particular, these findings add nuance to the proposed connection between estrogen synthesis and action in the temporal cortex and verbal memory in humans, indicating that this does not directly translate to the developing songbird brain. Historically, studies on rapid E2 signaling and learning have been largely focused on adults and hippocampal-dependent learning. Therefore, in addition to continuing to study the role of brain-derived estrogen signaling across a diverse range of animals ${ }^{121}$, it remains important to test its function across different ages ${ }^{122,123}$ and brain regions.

\section{Methods}

All methods and experiments were performed in accordance with relevant guidelines, regulations and with the approval of the University of Massachusetts Institutional Animal Care and Use Committee (protocols \#2017-0013 and 2016-0053).

Immunocytochemistry. Animals, perfusion, and sectioning. We first sought to confirm the presence of aromatase in NCM across development. While previous studies have assessed aromatase expression in the brains of developing songbird ${ }^{49-53}$, there is limited information on aromatase protein expression within NCM between the sensory and sensorimotor periods, which represents a critical transition in auditory response and neuroestrogen sensitivity ${ }^{40}$. Male juvenile zebra finches $(n=6)$ were selected from mixed-sex breeding aviaries maintained on a 14:10 light:dark cycle. Male sensorimotor subjects $(n=3 ; 65,71$, and $71 \mathrm{dph})$ were identified by their sexually dimorphic plumage (orange cheek feathers; brown and black badge feathers). Sensory-aged male subjects without dimorphic plumage $(n=3 ; 20,26$, and $34 \mathrm{dph})$ were identified by PCR (see Sex Determination below). All subjects were obtained from our breeding colony and were exposed to adult song up until the day of the perfusion. Birds were euthanized via anesthetic overdose (isoflurane) and transcardially perfused with 20-30 mL of $0.1 \mathrm{M}$ phosphate buffer saline (PBS) followed by $35 \mathrm{~mL}$ of $4 \%$ paraformaldehyde (PFA). After perfusion, brains were extracted and placed into $4 \%$ PFA for 24 hours at $4{ }^{\circ} \mathrm{C}$. Brains were then transferred to a $30 \%$ sucrose- $0.1 \mathrm{M}$ PBS solution for $24-48$ hours at $4^{\circ} \mathrm{C}$. Once fixed, brains were submerged in an opaque tissue-embedding medium (O.C.T. compound; Tissue-Plus; Fisher Health-Care) and frozen at $-80^{\circ} \mathrm{C}$. Brains were thawed on wet ice on the day of sectioning and hemisected using a razor blade to allow us to carefully distinguish hemispheres. Brains were sectioned at $35 \mu \mathrm{m}$ in the sagittal plane at $-20^{\circ} \mathrm{C}$ using a cryostat (Leica CM3050 S). Each hemisphere was separately collected into two series for lateral sections, and four series for medial sections. Medial sections were determined by the emergence of cerebellum. Sectioned tissue was placed in cryoprotectant medium in 12-well plates, which was wrapped with Parafilm and stored at $-20^{\circ} \mathrm{C}$ until immunocytochemistry.

Antibodies. Antibodies and dilutions for aromatase and parvalbumin were identical to those used in Ikeda, et $a l^{55}$. Briefly, we used a polyclonal anti- aromatase primary antibody raised in rabbit (1:2,000; a generous gift from Dr. Colin Saldanha), and a monoclonal anti-parvalbumin primary antibody raised in mouse (1:10,000; Millipore MAB1572; RRID: AB_2174013). Secondary antibodies included goat anti-rabbit Alexa 488 (1:500; Thermo Fisher Scientific Inc.), and goat anti-mouse Alexa 647 (1:100; Thermo Fisher Scientific Inc.).

Procedure. Brain sections were first manually washed $3 \times$ in $0.1 \mathrm{M} \mathrm{PB}$, followed by $3 \times 15$-minute washes in $0.1 \mathrm{M}$ PB on a plate shaker, followed by a 2-hour incubation at room temperature with $10 \%$ normal goat serum (Vector) in $0.3 \%$ PBT. Tissue was then transferred to a $10 \%$ normal goat serum $-0.3 \%$ PBT solution containing the primary antibodies and incubated at room temperature for 60 minutes. Afterwards, plates were tightly wrapped in parafilm and placed on an orbital shaker in a cold room at $4{ }^{\circ} \mathrm{C}$ for 48 hours. On day 3, tissue was washed $3 \times$ 15 minutes in $0.1 \%$ PBT before being transferred to the secondary antibody-containing solution made in $0.3 \%$ PBT for 60 minutes. At this point, tissue was kept in the dark to prevent any fluorescent bleaching. Tissue was washed again $3 \times 10$ minutes in $0.1 \%$ PBT, and finally transferred to $0.1 \mathrm{M} \mathrm{PB}$, wrapped in parafilm, and stored at $4^{\circ} \mathrm{C}$. Several days later, tissue was slide mounted, covered with ProLong Diamond Antifade Mountant with DAPI (Thermo Fisher Scientific Inc.), cover slipped, and placed in an opaque slide box and stored at $4^{\circ} \mathrm{C}$.

Confocal imaging. Fluorescently-labelled tissue was imaged using a confocal microscope (Nikon A1 Resonant Confocal) with NIS-Elements imaging software. The laser strength and gain were determined independently for each antibody/fluorescent channel of interest. Once the levels were determined, the same setting was applied across all sections per fluorescent channel. NCM was located anatomically by the presence of cerebellum and the absence of aromatase-rich nucleus taenia (TnA; lateral boundary of NCM) as in previous studies ${ }^{55}$. An overview/reference image at $10 \mathrm{x}$ was obtained for each section followed by subregion (dNCM and vNCM) z-stacks obtained at $60 \times(1 \mu \mathrm{m} \mathrm{z}$-steps for $15 \mu \mathrm{m})$. 
Image analysis. An experimenter blind to subjects' ages and hemisphere quantified the total number immunostained cells for each fluorescent channel using ImageJ $1.52 \mathrm{~h}^{124}$. Cells were quantified at each $\mathrm{z}$-plane, with a companion collapsed $z$-stack image used as reference to ensure the same cell was not counted twice. We measured immunopositive-neurons two ways. Initially, we quantified aromatase and parvalbumin immunopositive-cells by calculating their expression as a percentage of DAPI-positive nuclei (cell count) to normalize for relative cell density across sections and subjects (e.g. Aromatase + cells $\%$ of DAPI $=$ total \# of aromatase + cells/total \# of DAPI + cells). Additionally, we also quantified cell density relative to image volume to provide a more standardized report of its expression using the following equation:

$$
\text { Cell density }\left(\mathrm{mm}^{3}\right)=\frac{\text { total\# of immunopositive }- \text { cells }}{\text { image volume }}
$$

Juvenile song learning. Animals. Juvenile male zebra finches (Taeniopygia guttata) were obtained from our breeding aviaries $(N=34 ; n=6$ for systemic experiments; $n=28$ for microdialysis experiments. See Fig. 2 for the experimental timeline). Nest boxes with an active clutch of young zebra finches $(<10 \mathrm{dph})$ were observed to identify the putative mother. Once identified, the mother, offspring, and their nest box were placed in a cage within a sound-attenuation chamber (Eckel Acoustics), either as a single-family group, or, in a few rare instances, two adjacent cages of females with siblings were placed in the same chamber. Some breeding pairs were also isolated before laying a clutch $(n=3)$. In these instances, the adult male was left in the sound-attenuation chamber until the fledglings were $\sim 13 \mathrm{dph}$. The remaining fledgling were removed from the breeding colony by $13 \mathrm{dph}$ (range $=5-17 \mathrm{dph}$ ), which is well before the putative opening of the critical period for song learning $(\sim 20-25$ $\mathrm{dph})^{125-129}$. Birds were confirmed to be male via sex determination PCR at $\sim 22 \mathrm{dph}$. By $\sim 30 \mathrm{dph}$, most birds were isolated from their siblings and mom (range $=29-39 \mathrm{dph} ; n=2$ birds that were $>38 \mathrm{dph}$; most birds were 29-31 $\mathrm{dph}$ ) and placed in a new cage and sound-attenuation chamber along with an unrelated adult companion female. An omnidirectional microphone (Countryman) was placed in the chamber and song was continuously recorded for the remainder of the experiment using Sound Analysis Pro ${ }^{62}$.

For microdialysis subjects, a total of 20 birds were successfully treated with FAD or aCSF ( $n=5$ subjects per hemisphere per treatment). An additional eight subjects experienced non-health related technical issues during microdialysis (e.g. clogged microdialysis probe) that resulted in them being prematurely disconnected but retained as surgery control subjects ('cannula'-only subjects). One of these failed microdialysis subjects was deprived of any tutoring or adult male song until after $131 \mathrm{dph}$ and served as an isolate control subject.

Timeline. Systemic: For systemically-treated subjects, birds were co-housed with an adult companion female throughout the entire experiment. Tutoring began at $40 \mathrm{dph}$ (see Tutoring regiment) and was immediately followed by oral administration of the assigned treatment. Tutoring continued every other day for 20 days (i.e. 10 days of total tutoring), ending at $60 \mathrm{dph}$. Peripheral FAD treatment suppresses E2 synthesis for up to 48 hours $^{58}$. Thus, there was one 'washout' day without any treatments between each tutoring session. Birds were returned to group housing at $131 \mathrm{dph}$, and after at least 6 weeks ( $\sim 196 \mathrm{dph})$, were re-captured to record song and terminal electrophysiology recordings.

Microdialysis: Guide cannulae were unilaterally implanted in NCM several days after being initially isolated with a companion female. Several days following surgery, birds were connected to the microdialysis apparatus in a new sound-attenuation chamber without any companion birds. One day later, daily tutoring began for two to three days. After the last tutor session, birds were disconnected from the microdialysis setup and placed in a sound-attenuation chamber with an adult female companion bird in an adjacent cage. Companion females were switched every two weeks to reduce any potential influence on the pupil's developing song ${ }^{99}$. Birds were returned to a group setting (all-male aviary in breeding room, or in a mixed-sex sound-attenuation chamber in same-sex cage) at $131 \mathrm{dph}$. After at least 6 weeks had elapsed, birds were returned to a sound-attenuation chamber for follow-up song recording and subsequent electrophysiology experiments. After electrophysiology recordings, birds were sacrificed, and brains were extracted for future sectioning and histological examination.

Sex determination. Zebra finches begin to develop sexually dimorphic plumage at $\sim 30-40 \mathrm{dph}$. Therefore, we used established methods ${ }^{130}$ as we have previously described ${ }^{40,49}$ to determine juvenile birds' sex. Briefly, DNA for sex determination PCR was extracted from whole blood obtained from the ulnar vein typically at $\sim 22 \mathrm{dph}$ (median age $=22 \mathrm{dph}$; range $=18-30 \mathrm{dph}$ ). Identified males were retained for the experiment, whereas females were returned to their original breeding aviary along with their mother once the youngest male fledgling reached $\sim 30 \mathrm{dph}$.

Pharmacological inhibition of aromatase. For systemic experiments, birds were fed $30 \mu \mathrm{L}$ of either saline $(0.9 \%$ $\mathrm{NaCl}$ in $\left.{ }_{\mathrm{dd}} \mathrm{H} 20\right)$ or FAD $(1 \mathrm{mg} / \mathrm{mL}$ in $0.9 \% \mathrm{NaCl})$ immediately following tutoring cessation. This dose is similar to previous studies that demonstrate significantly reduced aromatase activity and/or estradiol levels in zebra fin ches $^{24,38,50,58,131}$. Microdialysis subjects were retrodialyzed with artificial cerebrospinal fluid (aCSF) and $100 \mu \mathrm{M}$ FAD in aCSF prepared as in previous experiments ${ }^{38,49,78,79}$.

In Vivo Microdialysis. A unilateral CMA-7 microdialysis guide cannula with obdurator (CMA Microdialysis, CMA 7, ref. no. P000138) was implanted several days after isolation with a companion female (median age $=39$ $\mathrm{dph}$; range $=35-47 \mathrm{dph}$ ), as in previous studies ${ }^{49,79,132}$. Birds were food deprived 30 minutes prior to surgery, and then received an intramuscular injection of Equithesin (30-40 $\mu \mathrm{L}$, typically). Twenty minutes later, birds were swaddled in a Kim wipe, and placed atop a heating pad and secured via ear bars at $45^{\circ}$ to our custom surgical 
stereotaxic apparatus (Herb Adams Engineering). Head feathers were removed and a $20 \mu \mathrm{L}$ subcutaneous injection of $2 \%$ lidocaine was administered underneath the scalp, which was subsequently resected to expose the outer layer of skull. The midsagittal sinus bifurcation (MSB) was then identified and used as a 0-point anatomical reference. A unilateral fenestra was then made over one hemisphere of NCM (coordinates: rostral $=1.20 \mathrm{~mm}$, lateral $= \pm 0.90 \mathrm{~mm}$ ), and the dura was carefully resected. A CMA-7 guide cannula with obdurator was then descended approximately $1.0 \mathrm{~mm}$ ventral into the proximate region of NCM (ventral range of NCM at this coordinate is $0.80-1.40 \mathrm{~mm}$ ). The guide cannula was secured using cyanoacrylate and dental cement, and the exposed scalp and incision area sealed with cyanoacrylate. Birds recovered on a heating pad in a cage with ad libitum food and water until awake, after which they were transferred back to their sound-attenuation chamber in a separate cage from the companion female.

Acute neural injury induces glial aromatase production in birds, with aromatase responses peaking at 72 hours, and persisting up to six weeks after insult ${ }^{133-135}$. To reduce the confound of injury-induced aromatase upregulation from the guide cannula surgery, birds were given at least three days to recover prior to starting microdialysis (median $=4$ days; range $=3-5$ days) to allow for injury-induced glial aromatase levels to subside ${ }^{136}$.

After the recovery period, birds were connected to the microdialysis apparatus in a new sound-attenuation chamber. The obdurator was replaced with a CMA-7 microdialysis probe $(1 \mathrm{~mm}$ membrane length, CMA Microdialysis, ref. no. P000082), which was then connected to a dual-channel microdialysis swivel (375/D/22QM; Instech Labs) via fluorinated ethylene propylene (FEP) inlet and outlet tubing. Once the bird was connected, aCSF was dialyzed at a rate of $2 \mu \mathrm{L} / \mathrm{min}$ by a syringe pump located outside of the chamber (PHD 1000, Harvard Apparatus). After being hooked-up, all birds were observed to ensure they were healthy as evidenced by eating, drinking, and the ability to comfortably navigate the cage. Dialysate samples were collected every hour during the day $(\sim 09: 00-\sim 18: 00 \mathrm{pm})$, yielding $\sim 120 \mu \mathrm{L}$ of dialysate per sample. Perfusate was dialyzed at a rate of $2 \mu \mathrm{L} /$ min for the entire duration of the microdialysis experiment. Several hours after the final tutor session, FEP tubing was disconnected and birds were returned to a sound-attenuation chamber in a separate cage alongside an adult companion female. As described in similar studies ${ }^{5}$, guide cannulae eventually detach after experiments as the skull develops and expands, without any obvious deleterious health effects, typically 12 days after the last day of microdialysis (range $=6-38$ days post-final microdialysis day; in one case, this did not occur until 154 days after microdialysis).

Tutoring regimen. All birds were naïve to song before the tutoring period. After tutoring, all birds were returned to an individual sound-attenuation chamber with an unrelated adult female companion in an adjacent cage. Including a companion female is atypical for most experimental studies of song learning in the lab, and there is some evidence to suggest that adult females may impact song development in juvenile male zebra finches ${ }^{137}$ and cowbirds ${ }^{138}$. However, we opted to include a companion female as isolating subjects is less naturalistic for zebra finches (a highly gregarious songbird), and likely a great deal more stressful for developing subjects.

Passive audiovisual tutoring playback. In an initial pilot experiment, we were curious whether an automated passive playback tutoring design would enable accurate song learning/imitation in adulthood, as used in other song tutoring studies ${ }^{49,60}$. Similar early isolation procedures as with the systemic and microdialysis subjects were used on a separate set of birds $(n=8)$. Otherwise unmanipulated subjects were isolated from their mother and siblings $\sim 37 \mathrm{dph}$, and daily tutoring began at $42 \mathrm{dph}$ until $47 \mathrm{dph}(5$ sessions total). Tutoring began at $\sim 10: 00$ each day and lasted for one hour. During the tutoring session, a 60-minute tutoring video was played on a USB-powered LCD monitor (Lilliput 7-in) alongside song broadcasted via an adjacent speaker (Sony; model \# SRS-TP1WHI). The video and song were obtained from an adult male zebra finch singing directed song to a female. At $48 \mathrm{dph}$, birds were reunited with an adult female companion and kept in isolation until $111 \mathrm{dph}$, after which time they were returned to a mixed-sex aviary. Song was recorded throughout the entirety of the experiment. Overall, birds tutored with passive audiovisual methods produced poor copies of the tutor song $(n=6$; mean \pm SEM; similarity $=41.09 \% \pm 0.07 \%$; range $=23.37-64.98 \%)$, likely due to zebra finches requiring active/ self-solicited learning (e.g. operant tutoring) and/or social instruction reviewed in ${ }^{3}$. Therefore, all remaining subjects were exposed to a hybrid live-tutoring with passive song playback of that tutor that yielded more reliable tutor song imitation.

Live tutoring with audio playback - systemic subjects. Audio visual tutoring methods did not yield successful tutor imitations. Therefore, we opted for a tutoring paradigm that included a live-male tutor alongside passive audio playback as in London and Clayton ${ }^{5}$. Unlike some songbird species that can learn song from passive audio playbacks e.g ${ }^{139,140}$., zebra finches require either operantly-evoked playbacks or social instruction ${ }^{3,69,141}$. We developed a tutor playback that combined passive audio playback alongside a live adult male. While operant playback has been used successfully to tutor zebra finches, we wanted to target the post-tutoring period with higher temporal precision. Operant training is pupil initiated and can span a long time period, whereas a controlled, timed playback allowed us to target the period immediately after training (i.e. the putative auditory memory consolidation period). To that end, we first identified a non-breeding adult male from our colony that was vocally active, and sang in the presence of an observer. The tutor was placed in a sound-attenuation chamber with an adult female and female-directed song was recorded, from which a 60-minute tutoring playback file was created. The same tutor playback and adult male was used for all systemically-treated subjects, as well as several of the microdialysis subjects $(n=22)$. After the original tutor perished, a new adult male was recruited, and a similar one-hour tutor playback file was created and presented to the remainder of subjects $(n=15)$.

The tutor playback file consisted of a 12-minute clip with 40 unique song bouts that was repeated five times. Each song bout contained 2-8 motifs, and included introductory notes. The 12-minute clip was assembled from 12 individual 1-minute blocks, where each block contained 30 seconds of song ( $4-5$ song bouts per song period, each separated by 5 seconds of silence) followed by 30 seconds of silence. The final tutoring playback file was 
amplified to $\sim 70 \mathrm{~dB}$ (A-weighted) and bandpass filtered at $0.3-15 \mathrm{kHz}$ (Adobe Audition), and played through a portable speaker (Sony, model\# SRS-TP1WHI) placed inside the sound-attenuation chamber.

The tutor was placed in an individual cage and kept in a sound-attenuation chamber with other adult zebra finches at least 24 hours before the day of tutoring. On the day of tutoring, an experimenter placed the tutor cage beside the pupil's cage. After a 10-minute acclimation period without any song playback, the tutoring playback recording began. Immediately after the end of the tutor playback file, the tutor was removed from the pupil's chamber.

Bioacoustic analysis. Automated song analysis. Percent similarity, accuracy, and \% sequence similarity was analyzed using SAP ${ }^{62}$. Ten motifs of the tutor song were each compared to ten motifs of each pupil's song from 130 dph using default settings for asymmetric mean values, yielding 100 comparisons per subject. Similar methods were used for measuring Wiener entropy (WE) and entropy variance (EV) across development in systemic subjects. As only half of the systemically-treated subjects produced song pre-tutoring $(n=3 ; 1$ FAD subject and 2 saline subjects), we averaged pre-tutoring WE and EV across all subjects to compare with relative to 49 dph, which was the first day all subjects produced song.

Manual song similarity analysis. In addition to automated song similarity methods, we also measured the number of tutor syllables copied by each subject and the quality of each copy. Coded and randomized motifs were qualitatively analyzed on a syllable-by-syllable basis as being either 'good', 'poor', or 'not available' relative to the tutor song by three experimenters blind to treatment conditions and subject identification. We confirmed that raters agreed across multiple dimensions by performing inter-rater reliability measurements using an unweighted Fleiss's Kappa. Raters were in excellent agreement in assessing syllable accuracy $(\mathrm{K}=0.563, p<0.001)$, assessing the syllables pupils were likely imitating $(\mathrm{K}=0.657, p<0.001)$, and on the total number of syllables copied from a tutor by a pupil $(\mathrm{K}=0.455, p<0.001)$. Moreover, raters' intra-reliability was similarity high: raters agreed on $60.46 \%$ of syllable accuracy, $65.12 \%$ on pupil syllables that reflect the tutor syllable, and $58.14 \%$ on both the accuracy and imitated syllable in the pupil's song. Further, raters' similarity scores were well-matched to the SAP measurements: there was a significant positive correlation between all raters visual similarity scoring and SAP's \% similarity measurement $(r(97)=0.75, p<0.001$; Supp. Fig. 2B).

Singing rate. An experimenter blind to treatment conditions measured the daily number of song bouts and their length for the entire pre-tutoring period (3-5 days pre-tutoring), tutoring period (10 days; tutor-off days), and every 5 days after the last day of tutoring until $130 \mathrm{dph}$ (14 days). An individual song bout was defined as being at least $1 \mathrm{~s}$ in total duration and considered unique if $500 \mathrm{~ms}$ of silence elapsed between singing periods. Song bouts were analyzed for one 3-hour period per analyzed day (14:00-17:00). These methods were adapted from previous studies measuring song rate ${ }^{142-145}$.

Adult song plasticity. In a subset of formerly microdialyzed birds $(n=23)$, we compared birds' own song at $130 \mathrm{dph}$ (putative closure of the critical period for song learning) and song after being exposed to other adult male song ( $>6$ weeks post-130 dph return). We used simple qualitative measurements to assess whether song had changed (either 'yes' or 'no' based on visual comparisons of several song files from each time point) instead of more thorough bioacoustic analyses as treatment did not appear to affect the likelihood of changing adult song (see Results), which was the main question of the experiments. Our visual assessment mainly asked: 1) did song crystallize (i.e. if it was previously variable across song bouts, was it now stable/consistent?); and 2) were any syllables added and/or dropped? This visual assessment protocol has several caveats, such as the inability to detect fine spectral changes. However, adult song plasticity was an incidental observation and not the main focus of our experiments, and as such, we did not pursue a more thorough/objective analysis.

Behavior. Female two-choice song phonotaxis. Female songbirds use song to evaluate a potential mate ${ }^{61,146,147}$. Therefore, in addition to measuring song similarity, we also tested whether less subtle song features were affected by treatment by measuring song preference in adult female zebra finches. A $13^{\prime \prime} \times 10^{\prime \prime}$ cage was placed in the center of a sound-attenuation chamber alongside speakers set on either side of it. Three ground-level perches were placed in the left- and right-most extreme side of the cage floor. A piece of cardboard cage matting was placed on the cage floor and divided into quarters with colored tape: left, left of middle, right of middle, and right. A non-breeding adult female zebra finch from our aviary $(N=12)$ was placed in the two-choice cage and isolated for $\sim 24$ hours before the playback experiment began to increase salience of the future song playback. On Day 2, a 30 min song file was presented starting at $\sim 13: 00$. The song file consisted of a 2-minute clip repeated 15 times. The first minute of the 2-minute clip contained adult song solely from one FAD or saline bird, whereas the second minute of the 2-minute clip contained song from only one bird of the opposite treatment condition. Each 1-minute clip included $5 \mathrm{~s}$ of song, followed by $5 \mathrm{~s}$ of silence, which was repeated 4 times ( $40 \mathrm{~s}$ total), and followed by $20 \mathrm{~s}$ of silence ( $60 \mathrm{~s}$ total) played on one side of the speakers. The 1-minute clip of the second bird was broadcasted on the opposite speaker in a similar manner. The same 2-minute clip was repeated 15 times (30 mins total). On Day 3, a different playback file was played at a similar time ( 13:00) with new song stimuli played on opposite speakers compared to Day 2 to account for potential side-bias (e.g. if FAD song was broadcasted on the left speaker on Day 2, a new FAD song was broadcasted on the right speaker on Day 3). Females were returned to the aviary after the cessation of Day 3 playbacks. Birds were excluded from analysis if they spent the entire time in the middle/neutral zone (one bird was excluded from analysis from both days, and another bird was excluded from just one day of analysis). Total time spent near either the FAD or saline side was measured. Additionally, a FAD preference ratio was calculated similar to Remage-Healey, et al. ${ }^{38}$ :

$$
\text { FAD Preference Ratio }=\frac{\sum \text { time spent near FAD speaker }}{\sum \text { time spent near FAD speaker }+\sum \text { time spent near saline speaker }}
$$


Microdialysis tutoring session behavior. Pupils who are more 'attentive' to the tutor during song learning sessions produce more similar copies of the tutor song in adulthood ${ }^{6,66}$. As such, we explored whether treatment affected pupils' behavior during tutoring sessions. Subjects were videotaped for 3 one-hour periods during each tutoring day, including: (1) the hour just prior to tutoring onset; (2) the tutoring period ( 70 mins; 10-minute acclimation period +60 min audio playback); and (3) the hour immediately after tutor offset. Three 10-minute clips per tutoring period for each subject were created for future behavioral scoring, including: (1) tutor acclimation period; (2) the beginning of tutor playback; and (3) 20-30 mins into the tutor playback period. The 10-min blocked videos (i.e., not the entire hour of tutoring session) were scored for numerous behaviors by an experimenter blind to subjects' treatment conditions using JWatcher ${ }^{148}$. Behaviors quantified included: events (eating; drinking; perch hops; grooming/preening; jumps; flights; feather ruffling; head scratching), and states (resting/ sleeping; tutor zone; outside of tutor zone; not in view). Tutor behavior also likely plays an important role on song learning. While tutors were observed vigorously singing during the playback/tutoring sessions, our recording design unfortunately precluded the ability to disentangle playback tutor song from live tutor song nor allow us to analyze tutor analyze behavior.

Electrophysiology. Surgery. As others have reported e.g. ${ }^{5}$, guide cannulae implanted during development eventually dissociate from the skull, and the wound heals normally (see Methods), which allowed us to perform electrophysiology recordings from formerly dialyzed birds in adulthood. Surgical methods for the electrophysiology experiments were similar to previous procedures ${ }^{39,40,149,150}$, the main difference being a lack of an implanted microdialysis probe in the current experiment. At least six weeks following birds being returned to the aviary (median age on day of surgery $=227 \mathrm{dph}$, range $=158-526 \mathrm{dph}$ ), birds were recaptured, placed in a cage with a companion adult female, and song was recorded. On the day of the surgery, birds were initially food deprived for 30 minutes. Afterwards, an intramuscular injection of Equithesin was administered, and 20 minutes later, birds were swaddled in a Kim wipe, and placed atop a heating pad and secured via ear bars to a custom surgical stereotaxic apparatus $\left(50^{\circ}\right.$ head angle). The bird's beak was opened and placed in a beak holder. Once the bird was secured, head feathers were removed, and a $20 \mu \mathrm{L}$ subcutaneous injection of $2 \%$ lidocaine was administered underneath the scalp, which was subsequently resected to expose the outer layer of skull. The MSB was then identified and used as our 0-point anatomical reference. A positioning needle was placed over the MSB, and adjusted to bilaterally mark NCM (rostral: $-1.4 \mathrm{~mm}$; lateral: $\pm 1.1 \mathrm{~mm}$ ) and HVC (lateral: $\pm 2.40 \mathrm{~mm}$ ). A piece of silver wire was inserted between the skull leaflets over the cerebellum to serve as a reference ground. A custom-fabricated metal head-post was then affixed above the beak and skull using dental cement and cyanoacrylate, followed by sealing the exposed scalp with cyanoacrylate. After surgery, birds were placed on heating pad within a recovery cage and provided with ad libitum food and water. Once birds awoke, they were returned to their sound-attenuation chamber in a separate cage from the companion female.

Anesthetized extracellular recordings. Extracellular, multiunit electrophysiological recordings were obtained from NCM and HVC in anesthetized subjects ( $n=21$ birds [aCSF $=8$ birds; FAD $=8$ birds; cannula $=5$ birds]; single-units $\mathrm{x}$ treatment $\mathrm{x}$ region: $\mathrm{aCSF}=20 \mathrm{HVC}$ units; 49 NCM units; FAD $=31$ HVC units; 48 NCM units; cannula $=14$ HVC units; 18 NCM units) using Spike2 (version 7.04, Cambridge Electronic Design) at a sampling rate of $16.67 \mathrm{kHZ}$, bandpass-filtered at $0.3-5 \mathrm{kHz}$. On the day of the experiment, birds were starved for 30 mins, followed by three intramuscular injections of $20 \%$ urethane on alternating sides of pectoral muscle $(\sim 100 \mu \mathrm{L}$ total; $\sim 33 \mu \mathrm{L}$ per injection). Injections were administered every 45 minutes. Once anesthetized, birds were brought up to the recording room, wrapped in a Kim wipe, placed on a heating pad, and affixed to a custom head-post stereotaxic apparatus. The outer- and inner-leaflet of skull and dura over the HVC and NCM of one hemisphere was then removed. A drop of silicone oil was placed over the exposed brain to prevent the tissue from drying out. Individual carbon-fiber electrodes (CarboStar-1; Kation) were advanced into the proximate region of NCM and HVC based on: 1 ) anatomical location ( 0.80-1.40 $\mathrm{mm}$ ventral; and $\sim 0.50 \mathrm{~mm}$ ventral, respectively); and 2 ) characteristic spontaneous- and stimulus-evoked firing rates. In anesthetized adult songbirds, HVC preferentially responds to playbacks of birds' own song (BOS) $)^{151,152}$. As such, we played BOS along with other songs in our search stimuli set (see below) and used a combination of characteristic spontaneous activity and neural responses to BOS as an indication of placement within HVC. After a completed playback trial, electrodes were advanced $100-150 \mu \mathrm{m} \mathrm{dorsal} / \mathrm{ventral}$ along the same track, and, if the region-specific characteristic firing persisted, a new recording was obtained. Once a track was past anatomical limits and/or ceased to display characteristic firing patterns, an electrolytic lesion presented at the most recent site for future anatomical confirmation. After one hemisphere was complete, the contralateral hemisphere was exposed and recorded. At the end of the experiment, birds were rapidly decapitated, and their brains were extracted and placed in a $20 \%$ sucrose-formalin solution for future sectioning and histology. In addition to recording from successful subjects (i.e. aCSF and FAD treated subjects), we also recorded from subjects that whose microdialysis cannulae became non-functional during the tutoring experiment. We present these data as a visual comparison as surgery controls (noted as 'Cannula' subjects) but due to the variability for microdialysis failure in these subjects, we omitted them from our statistical model.

Auditory stimuli and playback. All stimuli were $\geq 2 \mathrm{~s} \mathrm{long}$, adjusted to $\sim 70 \mathrm{~dB}$ (A-weighted), and bandpass filtered to $0.3-15 \mathrm{kHz}$ (Adobe Audition). Two sets of stimuli were used during the recordings. A search set was composed of two unique conspecific songs (i.e. zebra finch; CON), birds' own song (BOS), reverse BOS (REV-BOS), and white noise (WN). The experimental set was composed of two novel CONs, BOS, REV-BOS, tutor's song (TUT), reverse TUT (REV-TUT), and WN. Search stimuli were presented manually by the experimenter to confirm putative NCM and HVC sites, whereas the experimental set were played automatically and randomized via a custom written script in Spike. For experimental playbacks, each stimulus was pseudorandomly played once per block, with a total of 20 blocks being presented for each playback period. Stimuli were separated by a $10 \mathrm{~s}$ inter-stimulus interval \pm 0 - $2 \mathrm{~s}$ of random time. 
Single-unit spike sorting. Individual single units were sorted using default parameters in Spike2 v.7.04, Cambridge Electronic Design; as $\mathrm{in}^{40}$. Units were retained for analysis if they: 1) were distinctly clustered in a principal component analysis space (apart from noise and other units);2) had an interspike interval of $>1 \mathrm{~ms}$; and 3 ) were auditory responsive by visual inspection of the peristimulus time histogram and raster plots.

Data analysis. Single-unit electrophysiology recordings were analyzed using similar methods as in Vahaba, et al. ${ }^{40}$. Briefly, spontaneous firing rates were defined as the total number of waveform events (spikes) occurring in a 2 -second period prior to the onset of an auditory stimulus, whereas stimulus-evoked firing was defined as the number of spikes during a 2-second window starting at the onset of an auditory stimulus. The total number of spikes per the total amount of stimulus presentations were divided by the number of stimulus iterations to yield firing rates $(\mathrm{Hz})$. Firing rates were also $\mathrm{z}$-transformed to normalize data and account for variability across subjects and units using the following equation:

$$
Z-\text { score }=\frac{\bar{S}-\bar{B}}{\sqrt{\operatorname{Var}(S)+\operatorname{Var}(B)-2 \operatorname{Covar}(S, B)}}
$$

where $S$ and $B$ represents the number of stimulus-evoked and spontaneous spikes, respectively; $\bar{S}$ and $\bar{B}$ represent the mean number of stimulus and spontaneous spikes for a given stimulus.

We also analyzed stimulus selectivity using $\mathrm{d}$ prime $\mathrm{d}^{\prime 153}$, a psychophysics metric of discriminability used for assessing neural responses to a given stimulus relative to a different stimulus ${ }^{39,73}$, e.g. ${ }^{154}$, using the following equation:

$$
d_{A-B}^{\prime}=\frac{2\left(R S\left[\text { STIM }_{A}\right]-R S\left[S T I M_{B}\right]\right)}{\sqrt{\sigma^{2}\left[S_{T I M}\right]+\sigma^{2}\left[\text { STIM }_{B}\right]}}
$$

where $R S$ is the response strength (mean stimulus-evoked firing rate subtracted from the mean spontaneous firing rate), $S T I M_{A}$ represents the focal stimulus of interest, $S T I M_{B}$ represents the relative stimulus to compare other stimuli to, and $\sigma^{2}$ is the RS variance for a given stimulus. WN was used as the comparison stimulus for NCM recordings, and CON1 for HVC recordings (see Results) as in similar experiments from our lab ${ }^{39}$.

Adult habituation experiment. Subjects. A separate set of otherwise untreated adult male zebra finches $(n=22)$ were removed from our single-sex aviary (median age on day of electrophysiology recording $=274 \mathrm{dph}$; all males at least $120 \mathrm{dph}$ ) and placed in a cage within a sound-attenuation chambers alongside an adult companion female while song was recorded using Sound Analysis Pro $^{62}$. Birds were kept in the same cage until the day of the surgery which typically occurred after 3 days of isolation (mode $=3$ days isolation pre-surgery; range $=0-6$ days).

Surgery. The surgery methods used for this experiment were nearly identical to the one above. The main difference was that the skull was exposed solely over both hemispheres of NCM. After bilateral marking of NCM (coordinates $=$ rostral: $-1.20 \mathrm{~mm}$; lateral $=+/-1.10 \mathrm{~mm}$ ), the outer and inner leaflets of skull were carefully removed, leaving the dura intact as much as possible. Following silver wire implantation, a silicone dural sealant (Kwik-Sil, World Precision Instruments [WPI]) was placed over the exposed skull.

Auditory Training \& Drug Administration. Awake birds were placed in a custom-fabricated restraint tube and brought into the recording room. After being secured to the head-post stereotaxic apparatus, birds were presented with a repeated training stimulus (TRAIN). TRAIN consisted of 200 iterations of a single adult male zebra finch song with a $12 \mathrm{~s}$ ISI. Each iteration of the TRAIN song consisted of two direct song motifs within one song bout, including intro notes. Training lasted 46 minutes in total. Immediately after the final song iteration, 100 $\mathrm{nL}$ of either artificial cerebrospinal fluid (aCSF) or $100 \mu \mathrm{M}$ FAD in aCSF were unilaterally administered into one hemisphere of NCM. This volume has been successfully used in previous studies and appears to disperse across the extent of $\mathrm{NCM}^{39,155}$. Injections were administered via pre-loaded glass micropipettes broken back to $\sim 24 \mu \mathrm{m}$ internal diameter. After a micropipette was descended at a ventral depth of $1.10 \mathrm{~mm}$, it was left in place for $>2$ minutes following injection to prevent dispersal of the solution. Following the 2-minute pipette acclimation period, the solution was dispensed via a pressure-injection using a Pneumatic PicoPump (PV830; World Precision Instruments). Immediately following the first injection, the contralateral hemisphere was administered the opposite treatment of the initial hemisphere (e.g. if first treatment $=\mathrm{FAD}$, then the contralateral hemisphere received aCSF). We opted for opposite treatments for each hemisphere to reduce between-subject variability. Following drug treatments, the exposed skull and dura was sealed with a lower tear-strength silicone adhesive (Kwik Cast), and then the bird was returned to his cage.

Electrophysiology. Awake, restrained birds were brought back to the recording room for electrophysiology recordings 6 or 20 hours after training. Birds were non-anesthetized as habituation is not typically observed in anesthetized songbirds ${ }^{38}$, but se $^{119}$. Parylene-coated tungsten electrodes ( 0.5 or $2 \mathrm{M} \Omega$; A-M Systems) were descended bilaterally into the approximate drug injection region from Training. Recordings were amplified using an A-M system amplifier and obtained through a connected 1401 board and Spike2 (CED). A set of stimuli were first presented to the bird search stimuli to confirm the recording site displayed NCM characteristic-like auditory responses. After site confirmation, experimental stimuli were presented to the bird while neural activity was continuously recorded. Each recording site was electrolytically lesioned following playback. Recording sites/ exposures were once again covered with silicone adhesive, and birds were either sacrificed via rapid decapitation immediately after recordings $(n=8)$ or $2-3$ days later $(n=15)$ to allow for lesion sites to become more 
pronounced and readily observable in sectioned tissue (e.g. allow time for gliosis). Extracted brains were placed in $20 \%$ sucrose-formalin for future sectioning and histological verification of recording and drug site via Nissl stain.

Auditory Stimuli \& Playback. All auditory stimuli were presented at $\sim 70 \mathrm{~dB}$. Search stimuli consisted of a unique set of non-familiar conspecific song not used in the experimental stimuli set. Experimental playback stimuli presented during neural recordings included the trained conspecific song (TRAIN) and its reverse (REV-TRAIN), three novel conspecifics (CON1, CON2, CON3) and one reversed (REV-CON3), bird's own song (BOS) and its reverse (REV-BOS), and white noise (WN). To ensure birds were unfamiliar with the song presented, several stimuli were graciously adapted from an online zebra finch song repository (http://people.bu.edu/timothyg/ song_website/). We also used two songs from birds in our own breeding colony as they had been removed long before the experiment began. Birds were presented with 25 consecutive iterations of each experimental stimulus with a 12 second ISI in blocks (e.g. $25 \mathrm{CON} 1$ playbacks, then $25 \mathrm{CON} 2$ playbacks, then $25 \mathrm{WN}$ playbacks, etc.), as in previous experiments e.g. ${ }^{42}$.

Analysis. Analyses were inspired from previous studies with minor changes e.g. ${ }^{42}$. Briefly, multi-unit recordings were analyzed root mean squared (RMS) in Spike2 for the stimulus and baseline period. The stimulus period included the entire duration of playback stimulus $+100 \mathrm{~ms}$ after offset, whereas the baseline period was defined as a $500 \mathrm{~ms}$ period preceding stimulus onset. Mean baseline RMS was derived across the entire recording period, whereas mean stimulus RMS was calculated for each individual stimulus. Data were filtered on a per trial (i.e. stimulus repetition) basis. First, any trial exceeding two-times the mean RMS for either baseline or stimulus RMS (separately) was excluded. After, any trial above/below 2.5 standard deviations was then excluded for both stimulus and baseline RMS. Finally, a grand mean baseline RMS (derived from the entire recording period; across stimuli) was subtracted from stimulus RMS values, yielding an adjusted RMS. Slope was derived from trials 1-25 using the $\operatorname{lm}($ ) function via the stats package in $\mathrm{R}$.

Statistical analysis. All statistical analyses were performed using $\mathrm{R}^{156}$ via RStudio ${ }^{157}$ using several packages, including: tidyverse; plyr; sciplot; irr; corrplot; data.table; and Hmisc. Histology data (\% DAPI; cell density) were analyzed using a two-way ANOVA (NCM subregion X phase). Song production counts were analyzed using a two-way ANOVA (treatment X time of day). Pearson's correlation was used to analyze changes in Wiener entropy relative to eventual song similarity at $130 \mathrm{dph}$. One-way ANOVAs were employed to assess systemic treatments effect on song learning outcomes (separate analyses for per cent similarity, sequential similarity, and accuracy). Female phonotaxis data were analyzed using two-way ANOVAs (treatment X trial day). For microdialyzed subjects, automated and manual song similarity analyses were analyzed using a two-way ANOVA (treatment X hemisphere). Inter-rater reliability for manual song similarity scoring was analyzed using an unweighted Fleiss's kappa. The comparison between automated (SAP) and manual (visual) song similarity was measured using Pearson's correlation. Tutoring behavior was analyzed using a mixed-effects ANOVA (tutoring day [within] X treatment [between]), and a correlation matrix adjusted for multiple comparisons (adjusted $\alpha=0.00048$ ). For behavioral analyses, we restricted our data to the first 10 minutes of tutoring for only days 1 and 2 to be consistent as some subjects received three days of tutoring. A chi-squared was used to compare distributions of adult song plasticity across treatment. For electrophysiology measurements, a three-way ANOVA was employed (treatment X recording hemisphere $\mathrm{X}$ stimulus). Finally, for adult habituation neural recordings, adaption slopes were compared using two-way ANOVAs (treatment type [aCSF/nothing vs. FAD] X stimulus type [familiar vs. trained]). All post hoc comparisons were performed using Tukey's honestly significant difference (HSD) test, and were corrected for multiple comparisons. $P$-values $<0.05$ were considered significant. Data from 'cannula' subjects were omitted from any statistical model and are plotted throughout the manuscript as a visual comparison (see Results).

\section{Data availability}

The datasets generated during and/or analyzed during the current study are available in the Center for Open Science OSF repository, https://osf.io/tz423/?view_only=b19cc6f965d440f68853251218251124.

Received: 6 August 2019; Accepted: 5 February 2020;

Published online: 27 February 2020

\section{References}

1. Petkov, C. I. \& Jarvis, E. D. Birds, primates, and spoken language origins: behavioral phenotypes and neurobiological substrates. Frontiers in evolutionary neuroscience 4, 12, https://doi.org/10.3389/fnevo.2012.00012 (2012).

2. Kuhl, P. K. Brain mechanisms in early language acquisition. Neuron 67, 713-727, https://doi.org/10.1016/j.neuron.2010.08.038 (2010).

3. Derégnaucourt, S. Birdsong learning in the laboratory, with especial reference to the song of the Zebra Finch (Taeniopygia guttata). Interaction Studies 12, 324-350 (2011).

4. Bolhuis, J. J. \& Moorman, S. Birdsong memory and the brain: in search of the template. Neurosci Biobehav Rev 50, 41-55, https:// doi.org/10.1016/j.neubiorev.2014.11.019 (2015).

5. London, S. E. \& Clayton, D. F. Functional identification of sensory mechanisms required for developmental song learning. Nat Neurosci 11, 579-586, https://doi.org/10.1038/nn.2103 (2008).

6. Ahmadiantehrani, S. \& London, S. E. Bidirectional manipulation of mTOR signaling disrupts socially mediated vocal learning in juvenile songbirds. Proc Natl Acad Sci USA 114, 9463-9468, https://doi.org/10.1073/pnas.1701829114 (2017).

7. Yanagihara, S. \& Yazaki-Sugiyama, Y. Auditory experience-dependent cortical circuit shaping for memory formation in bird song learning. Nat Commun 7, 11946, https://doi.org/10.1038/ncomms11946 (2016).

8. Gobes, S. M. \& Bolhuis, J. J. Birdsong memory: a neural dissociation between song recognition and production. Current biology: CB 17, 789-793, https://doi.org/10.1016/j.cub.2007.03.059 (2007).

9. Canopoli, A., Zai, A. \& Hahnloser, R. Lesions of a higher auditory brain area during a sensorimotor period do not impair birdsong learning. Matters, https://doi.org/10.19185/matters.201603000018 (2016). 
10. Canopoli, A., Zai, A. \& Hahnloser, R. Bilateral neurotoxic lesions in NCM before tutoring onset do not prevent successful tutor song learning. Matters, https://doi.org/10.19185/matters.201612000007 (2017).

11. Frick, K. M., Kim, J. \& Koss, W. A. Estradiol and hippocampal memory in female and male rodents. Curr Opin Behav Sci 23, 65-74, https://doi.org/10.1016/j.cobeha.2018.03.011 (2018).

12. Vierk, R. et al. Aromatase inhibition abolishes LTP generation in female but not in male mice. J Neurosci 32, 8116-8126, https:// doi.org/10.1523/JNEUROSCI.5319-11.2012 (2012).

13. Srivastava, D. P., Woolfrey, K. M. \& Penzes, P. Insights into rapid modulation of neuroplasticity by brain estrogens. Pharmacological reviews 65, 1318-1350, https://doi.org/10.1124/pr.111.005272 (2013).

14. Luine, V. N. Estradiol and cognitive function: past, present and future. Horm Behav 66, 602-618, https://doi.org/10.1016/j. yhbeh.2014.08.011 (2014).

15. Blaustein, J. D. Treatments for Breast Cancer That Affect Cognitive Function in Postmenopausal Women. Policy Insights from the Behavioral and Brain Sciences 4, 170-177, https://doi.org/10.1177/2372732217717271 (2017).

16. Bayer, J., Glascher, J., Finsterbusch, J., Schulte, L. H. \& Sommer, T. Linear and inverted U-shaped dose-response functions describe estrogen effects on hippocampal activity in young women. Nat Commun 9, 1220, https://doi.org/10.1038/s41467-018-03679-x (2018).

17. Barth, C. et al. In-vivo Dynamics of the Human Hippocampus across the Menstrual Cycle. Sci Rep 6, 32833, https://doi.org/10.1038/ srep32833 (2016).

18. Woolley, C. S. \& McEwen, B. S. Estradiol mediates fluctuation in hippocampal synapse density during the estrous cycle in the adult rat. J Neurosci 12, 2549-2554 (1992)

19. Woolley, C. S. Acute effects of estrogen on neuronal physiology. Annual review of pharmacology and toxicology 47, 657-680, https:// doi.org/10.1146/annurev.pharmtox.47.120505.105219 (2007)

20. Frick, K. M. Building a better hormone therapy? How understanding the rapid effects of sex steroid hormones could lead to new therapeutics for age-related memory decline. Behav Neurosci 126, 29-53, https://doi.org/10.1037/a0026660 (2012).

21. Packard, M. G. Posttraining estrogen and memory modulation. Horm Behav 34, 126-139, https://doi.org/10.1006/hbeh.1998.1464 (1998).

22. Packard, M. G. \& Teather, L. A. Intra-hippocampal estradiol infusion enhances memory in ovariectomized rats. Neuroreport 8 , 3009-3013 (1997).

23. Rensel, M. A., Ellis, J. M., Harvey, B. \& Schlinger, B. A. Sex, estradiol, and spatial memory in a food-caching corvid. Horm Behav 75, 45-54, https://doi.org/10.1016/j.yhbeh.2015.07.022 (2015).

24. Rensel, M. A., Salwiczek, L., Roth, J. \& Schlinger, B. A. Context-specific effects of estradiol on spatial learning and memory in the zebra finch. Neurobiol Learn Mem 100, 41-47, https://doi.org/10.1016/j.nlm.2012.12.005 (2013).

25. Bailey, D. J., Ma, C., Soma, K. K. \& Saldanha, C. J. Inhibition of hippocampal aromatization impairs spatial memory performance in a male songbird. Endocrinology 154, 4707-4714, https://doi.org/10.1210/en.2013-1684 (2013).

26. Bailey, D. J. et al. Hippocampal Aromatization Modulates Spatial Memory and Characteristics of the Synaptic Membrane in the Male Zebra Finch. Endocrinology 158, 852-859, https://doi.org/10.1210/en.2016-1692 (2017).

27. Bailey, D. J. \& Saldanha, C. J. The importance of neural aromatization in the acquisition, recall, and integration of song and spatial memories in passerines. Horm Behav, https://doi.org/10.1016/j.yhbeh.2015.06.007 (2015).

28. Tuscher, J. J. et al. Inhibition of local estrogen synthesis in the hippocampus impairs hippocampal memory consolidation in ovariectomized female mice. Horm Behav 83, 60-67, https://doi.org/10.1016/j.yhbeh.2016.05.001 (2016).

29. Korol, D. L. \& Pisani, S. L. Estrogens and cognition: Friends or foes?: An evaluation of the opposing effects of estrogens on learning and memory. Horm Behav 74, 105-115, https://doi.org/10.1016/j.yhbeh.2015.06.017 (2015).

30. Caras, M. L. Estrogenic modulation of auditory processing: a vertebrate comparison. Front Neuroendocrinol 34, 285-299, https:// doi.org/10.1016/j.yfrne.2013.07.006 (2013).

31. Caras, M. L. \& Remage-Healey, L. In Hearing and Hormones (eds Andrew H. Bass, Joseph A. Sisneros, Arthur N. Popper, \& Richard R. Fay) 77-99 (Springer International Publishing, 2016).

32. Zimmerman, M. E. et al. Endogenous estradiol is associated with verbal memory in nondemented older men. Brain Cogn 76, 158-165, https://doi.org/10.1016/j.bandc.2011.01.011 (2011).

33. Fernandez, G. et al. Menstrual cycle-dependent neural plasticity in the adult human brain is hormone, task, and region specific. J Neurosci 23, 3790-3795 (2003)

34. Anthoni, H. et al. The aromatase gene CYP19A1: several genetic and functional lines of evidence supporting a role in reading, speech and language. Behavior genetics 42, 509-527, https://doi.org/10.1007/s10519-012-9532-3 (2012).

35. Wermke, K., Hain, J., Oehler, K., Wermke, P. \& Hesse, V. Sex hormone influence on human infants's sound characteristics: melody in spontaneous crying. Biology letters 10, 20140095, https://doi.org/10.1098/rsbl.2014.0095 (2014).

36. Schaadt, G., Hesse, V. \& Friederici, A. D. Sex hormones in early infancy seem to predict aspects of later language development. Brain and language 141, 70-76, https://doi.org/10.1016/j.bandl.2014.11.015 (2015).

37. Vahaba, D. M. \& Remage-Healey, L. Neuroestrogens rapidly shape auditory circuits to support communication learning and perception: Evidence from songbirds. Horm Behav, https://doi.org/10.1016/j.yhbeh.2018.03.007 (2018).

38. Remage-Healey, L., Coleman, M. J., Oyama, R. K. \& Schlinger, B. A. Brain estrogens rapidly strengthen auditory encoding and guide song preference in a songbird. Proc Natl Acad Sci USA 107, 3852-3857, https://doi.org/10.1073/pnas.0906572107 (2010).

39. Remage-Healey, L. \& Joshi, N. R. Changing neuroestrogens within the auditory forebrain rapidly transform stimulus selectivity in a downstream sensorimotor nucleus. J Neurosci 32, 8231-8241, https://doi.org/10.1523/JNEUROSCI.1114-12.2012 (2012).

40. Vahaba, D. M., Macedo-Lima, M. \& Remage-Healey, L. Sensory Coding and Sensitivity to Local Estrogens Shift during Critical Period Milestones in the Auditory Cortex of Male Songbirds. eNeuro 4, https://doi.org/10.1523/ENEURO.0317-17.2017 (2017).

41. Silverin, B., Baillien, M., Foidart, A. \& Balthazart, J. Distribution of aromatase activity in the brain and peripheral tissues of passerine and nonpasserine avian species. Gen Comp Endocrinol 117, 34-53, https://doi.org/10.1006/gcen.1999.7383 (2000).

42. Yoder, K. M., Lu, K. \& Vicario, D. S. Blocking estradiol synthesis affects memory for songs in auditory forebrain of male zebra finches. Neuroreport 23, 922-926, https://doi.org/10.1097/WNR.0b013e3283588b61 (2012).

43. Weichel, K., Schwager, G., Heid, P., Güttinger, H. R. \& Pesch, A. Sex Differences in Plasma Steroid Concentrations and Singing Behaviour during Ontogeny in Canaries (Serinus canaria). Ethology 73, 281-294, https://doi.org/10.1111/j.1439-0310.1986. tb00810.x (1986).

44. Marler, P., Peters, S., Ball, G. F., Dufty, A. M. Jr. \& Wingfield, J. C. The role of sex steroids in the acquisition and production of birdsong. Nature 336, 770-772, https://doi.org/10.1038/336770a0 (1988).

45. Marler, P., Peters, S. \& Wingfield, J. Correlations between song acquisition, song production, and plasma levels of testosterone and estradiol in sparrows. J Neurobiol 18, 531-548, https://doi.org/10.1002/neu.480180605 (1987).

46. Pröve, E. In Hormones and behaviour in higher vertebrates (eds J. Balthazart, E. Pröve, \& R. Gilles) (Springer-Verlag, 1983).

47. Quast, A., Hesse, V., Hain, J., Wermke, P. \& Wermke, K. Baby babbling at five months linked to sex hormone levels in early infancy. Infant Behav Dev 44, 1-10, https://doi.org/10.1016/j.infbeh.2016.04.002 (2016).

48. Acharya, K. D. \& Veney, S. L. Characterization of the G-protein-coupled membrane-bound estrogen receptor GPR30 in the zebra finch brain reveals a sex difference in gene and protein expression. Dev Neurobiol 72, 1433-1446, https://doi.org/10.1002/ dneu.22004 (2011). 
49. Chao, A., Paon, A. \& Remage-Healey, L. Dynamic variation in forebrain estradiol levels during song learning. Dev Neurobiol 75, 271-286, https://doi.org/10.1002/dneu.22228 (2015).

50. Saldanha, C. J. et al. Distribution and regulation of telencephalic aromatase expression in the zebra finch revealed with a specific antibody. J Comp Neurol 423, 619-630 (2000).

51. Saldanha, C. J., Clayton, N. S. \& Schlinger, B. A. Androgen metabolism in the juvenile oscine forebrain: a cross-species analysis at neural sites implicated in memory function. J Neurobiol 40, 397-406 (1999).

52. Jacobs, E. C., Arnold, A. P. \& Campagnoni, A. T. Developmental regulation of the distribution of aromatase- and estrogenreceptor- mRNA-expressing cells in the zebra finch brain. Developmental neuroscience 21, 453-472, 17413 (1999).

53. Vockel, A., Prove, E. \& Balthazart, J. Changes in the activity of testosterone-metabolizing enzymes in the brain of male and female zebra finches during the post-hatching period. Brain Res 463, 330-340 (1988).

54. Tremblay, R., Lee, S. \& Rudy, B. GABAergic Interneurons in the Neocortex: From Cellular Properties to Circuits. Neuron 91, 260-292, https://doi.org/10.1016/j.neuron.2016.06.033 (2016).

55. Ikeda, M. Z., Krentzel, A. A., Oliver, T. J., Scarpa, G. B. \& Remage-Healey, L. Clustered organization and region-specific identities of estrogen-producing neurons in the forebrain of Zebra Finches (Taeniopygia guttata). J Comp Neurol 525, 3636-3652, https://doi. org/10.1002/cne.24292 (2017).

56. Hensch, T. K. Critical period plasticity in local cortical circuits. Nature reviews. Neuroscience 6, 877-888, https://doi.org/10.1038/ nrn1787 (2005).

57. Balmer, T. S., Carels, V. M., Frisch, J. L. \& Nick, T. A. Modulation of perineuronal nets and parvalbumin with developmental song learning. J Neurosci 29, 12878-12885, https://doi.org/10.1523/JNEUROSCI.2974-09.2009 (2009).

58. Wade, J., Schlinger, B. A., Hodges, L. \& Arnold, A. P. Fadrozole: a potent and specific inhibitor of aromatase in the zebra finch brain. Gen Comp Endocrinol 94, 53-61, https://doi.org/10.1006/gcen.1994.1059 (1994).

59. Alward, B. A. et al. Aromatase inhibition rapidly affects in a reversible manner distinct features of birdsong. Sci Rep 6, 32344, https://doi.org/10.1038/srep32344 (2016).

60. Deshpande, M., Pirlepesov, F. \& Lints, T. Rapid encoding of an internal model for imitative learning. Proc Biol Sci 281, 20132630, https://doi.org/10.1098/rspb.2013.2630 (2014).

61. Zann, R. A. The zebra finch: a synthesis of field and laboratory studies. (Oxford University Press, 1996).

62. Tchernichovski, O., Nottebohm, F., Ho, C. E., Pesaran, B. \& Mitra, P. P. A procedure for an automated measurement of song similarity. Anim Behav 59, 1167-1176, https://doi.org/10.1006/anbe.1999.1416 (2000).

63. Borror, D. J. \& Reese, C. R. The Analysis of Bird Songs by Means of a Vibralyzer. The Wilson Bulletin 65, 276 (1953)

64. Thorpe, W. H. The process of song-learning in the chaffinch as studied by means of the spectrograph. Nature 173, 465-469, https:// doi.org/10.1038/173465a0 (1954).

65. Eales, L. A. Song learning in zebra finches: some effects of song model availability on what is learnt and when. Anim Behav 33, 1293-1300 (1985).

66. Chen, Y., Matheson, L. E. \& Sakata, J. T. Mechanisms underlying the social enhancement of vocal learning in songbirds. Proc Natl Acad Sci USA 113, 6641-6646, https://doi.org/10.1073/pnas.1522306113 (2016).

67. Sommer, T. et al. Effects of the experimental administration of oral estrogen on prefrontal functions in healthy young women. Psychopharmacology, https://doi.org/10.1007/s00213-018-5061-y (2018).

68. Morrison, R. G. \& Nottebohm, F. Role of a telencephalic nucleus in the delayed song learning of socially isolated zebra finches. J Neurobiol 24, 1045-1064, https://doi.org/10.1002/neu.480240805 (1993).

69. Deregnaucourt, S., Poirier, C., Kant, A. V., Linden, A. V. \& Gahr, M. Comparisons of different methods to train a young zebra finch (Taeniopygia guttata) to learn a song. Journal of physiology, Paris 107, 210-218, https://doi.org/10.1016/j.jphysparis.2012.08.003 (2013).

70. Jones, A. E., TenCate, C. \& Slater, P. J. B. Early experience and plasticity of song in adult male zebra finches (Taeniopygia guttata). Journal of Comparative Psychology 110, 354-369 (1996).

71. Picciotto, M. R. Recommendations for the Design and Analysis of In Vivo Electrophysiology Studies. J Neurosci 38, 5837-5839, https://doi.org/10.1523/JNEUROSCI.1480-18.2018 (2018).

72. Adret, P., Meliza, C. D. \& Margoliash, D. Song tutoring in presinging zebra finch juveniles biases a small population of higher-order song-selective neurons toward the tutor song. J Neurophysiol 108, 1977-1987, https://doi.org/10.1152/jn.00905.2011 (2012).

73. Moseley, D. L., Joshi, N. R., Prather, J. F., Podos, J. \& Remage-Healey, L. A neuronal signature of accurate imitative learning in wildcaught songbirds (swamp sparrows, Melospiza georgiana). Sci Rep 7, 17320, https://doi.org/10.1038/s41598-017-17401-2 (2017).

74. Vallentin, D., Kosche, G., Lipkind, D. \& Long, M. A. Inhibition protects acquired song segments during vocal learning in zebra finches. Science 351, 267-271, https://doi.org/10.1126/science.aad3023 (2016).

75. Volman, S. F. Development of neural selectivity for birdsong during vocal learning. J Neurosci 13, 4737-4747 (1993)

76. Prather, J. F., Peters, S., Nowicki, S. \& Mooney, R. Precise auditory-vocal mirroring in neurons for learned vocal communication. Nature 451, 305-310, https://doi.org/10.1038/nature06492 (2008).

77. Pawlisch, B. A. \& Remage-Healey, L. Neuroestrogen signaling in the songbird auditory cortex propagates into a sensorimotor network via an 'interface' nucleus. Neuroscience 284, 522-535, https://doi.org/10.1016/j.neuroscience.2014.10.023 (2015).

78. Remage-Healey, L., Dong, S. M., Chao, A. \& Schlinger, B. A. Sex-specific, rapid neuroestrogen fluctuations and neurophysiological actions in the songbird auditory forebrain. J Neurophysiol 107, 1621-1631, https://doi.org/10.1152/jn.00749.2011 (2012).

79. Remage-Healey, L., Maidment, N. T. \& Schlinger, B. A. Forebrain steroid levels fluctuate rapidly during social interactions. Nat Neurosci 11, 1327-1334, https://doi.org/10.1038/nn.2200 (2008).

80. Stripling, R., Kruse, A. A. \& Clayton, D. F. Development of song responses in the zebra finch caudomedial neostriatum: role of genomic and electrophysiological activities. J Neurobiol 48, 163-180 (2001).

81. Gentner, T. Q., Hulse, S. H. \& Ball, G. F. Functional differences in forebrain auditory regions during learned vocal recognition in songbirds. J Comp Physiol A Neuroethol Sens Neural Behav Physiol 190, 1001-1010, https://doi.org/10.1007/s00359-004-0556-x (2004).

82. Eda-Fujiwara, H. et al. Localized brain activation related to the strength of auditory learning in a parrot. PLoS One 7, e38803, https://doi.org/10.1371/journal.pone.0038803 (2012).

83. Sanford, S. E., Lange, H. S. \& Maney, D. L. Topography of estradiol-modulated genomic responses in the songbird auditory forebrain. Dev Neurobiol 70, 73-86, https://doi.org/10.1002/dneu.20757 (2010).

84. Phillmore, L. S., Bloomfield, L. L. \& Weisman, R. G. Effects of songs and calls on ZENK expression in the auditory telencephalon of field- and isolate-reared black capped chickadees. Behav Brain Res 147, 125-134 (2003).

85. Avey, M. T. et al. ZENK activation in the nidopallium of black-capped chickadees in response to both conspecific and heterospecific calls. PLoS One 9, e100927, https://doi.org/10.1371/journal.pone.0100927 (2014).

86. Thompson, J. V. \& Gentner, T. Q. Song recognition learning and stimulus-specific weakening of neural responses in the avian auditory forebrain. J Neurophysiol 103, 1785-1797, https://doi.org/10.1152/jn.00885.2009 (2010).

87. Matragrano, L. L. et al. Rapid effects of hearing song on catecholaminergic activity in the songbird auditory pathway. PLoS One 7, e39388, https://doi.org/10.1371/journal.pone.0039388 (2012).

88. Cornez, G. et al. Timing of perineuronal net development in the zebra finch song control system correlates with developmental song learning. Proc Biol Sci 285, https://doi.org/10.1098/rspb.2018.0849 (2018). 
89. Walters, M. J. \& Harding, C. F. The effects of an aromatization inhibitor on the reproductive behavior of male zebra finches. Horm Behav 22, 207-218 (1988).

90. Remage-Healey, L., Oyama, R. K. \& Schlinger, B. A. Elevated aromatase activity in forebrain synaptic terminals during song. J Neuroendocrinol 21, 191-199, https://doi.org/10.1111/j.1365-2826.2009.01820.x (2009).

91. Ikebuchi, M., Hasegawa, T. \& Bischof, H. J. Amygdala and socio-sexual behavior in male zebra finches. Brain Behav Evol 74, 250-257, https://doi.org/10.1159/000264660 (2009).

92. Korsia, S. \& Bottjer, S. W. Chronic testosterone treatment impairs vocal learning in male zebra finches during a restricted period of development. J Neurosci 11, 2362-2371 (1991).

93. Whaling, C. S., Nelson, D. A. \& Marler, P. Testosterone-induced shortening of the storage phase of song development in birds interferes with vocal learning. Dev Psychobiol 28, 367-376, https://doi.org/10.1002/dev.420280703 (1995).

94. Bottjer, S. W. \& Johnson, F. Circuits, hormones, and learning: vocal behavior in songbirds. J Neurobiol 33, 602-618 (1997).

95. Livingston, F. S. \& Mooney, R. Androgens and isolation from adult tutors differentially affect the development of songbird neurons critical to vocal plasticity. J Neurophysiol 85, 34-42 (2001).

96. Adkins-Regan, E., Abdelnabi, M., Mobarak, M. \& Ottinger, M. A. Sex steroid levels in developing and adult male and female zebra finches (Poephila guttata). Gen Comp Endocrinol 78, 93-109 (1990).

97. Frick, K. M. Molecular mechanisms underlying the memory-enhancing effects of estradiol. Horm Behav 74, 4-18, https://doi. org/10.1016/j.yhbeh.2015.05.001 (2015).

98. Cornil, C. A., Taziaux, M., Baillien, M., Ball, G. F. \& Balthazart, J. Rapid effects of aromatase inhibition on male reproductive behaviors in Japanese quail. Horm Behav 49, 45-67, https://doi.org/10.1016/j.yhbeh.2005.05.003 (2006).

99. Carouso-Peck, S. \& Goldstein, M. H. Female Social Feedback Reveals Non-imitative Mechanisms of Vocal Learning in Zebra Finches. Current biology: CB 29, 631-636 e633, https://doi.org/10.1016/j.cub.2018.12.026 (2019).

100. Williams, H. Models for song learning in the zebra finch: Fathers or others? Anim Behav 39, 745-757, https://doi.org/10.1016/ S0003-3472(05)80386-0 (1990).

101. Adret, P. Vocal imitation in blindfolded zebra finches (Taeniopygia guttata) is facilitated in the presence of a non-singing conspecific female. Journal of Ethology 22, 29-35, https://doi.org/10.1007/s10164-003-0094-y (2004).

102. Nick, T. A. \& Konishi, M. Neural auditory selectivity develops in parallel with song. J Neurobiol 62, 469-481, https://doi. org/10.1002/neu.20115 (2005).

103. Nick, T. A. \& Konishi, M. Neural song preference during vocal learning in the zebra finch depends on age and state. J Neurobiol 62, 231-242, https://doi.org/10.1002/neu.20087 (2005).

104. Prather, J. F., Peters, S., Nowicki, S. \& Mooney, R. Persistent representation of juvenile experience in the adult songbird brain. J Neurosci 30, 10586-10598, https://doi.org/10.1523/JNEUROSCI.6042-09.2010 (2010).

105. Roberts, T. F., Gobes, S. M., Murugan, M., Olveczky, B. P. \& Mooney, R. Motor circuits are required to encode a sensory model for imitative learning. Nat Neurosci 15, 1454-1459, https://doi.org/10.1038/nn.3206 (2012).

106. Dave, A. S. \& Margoliash, D. Song replay during sleep and computational rules for sensorimotor vocal learning. Science 290 , $812-816(2000)$

107. Soyman, E. \& Vicario, D. S. Principles of auditory processing differ between sensory and premotor structures of the songbird forebrain. J Neurophysiol 117, 1266-1280, https://doi.org/10.1152/jn.00462.2016 (2017).

108. Stripling, R., Volman, S. F. \& Clayton, D. F. Response modulation in the zebra finch neostriatum: relationship to nuclear gene regulation. J Neurosci 17, 3883-3893 (1997).

109. Grace, J. A., Amin, N., Singh, N. C. \& Theunissen, F. E. Selectivity for conspecific song in the zebra finch auditory forebrain. J Neurophysiol 89, 472-487, https://doi.org/10.1152/jn.00088.2002 (2003).

110. Janata, P. \& Margoliash, D. Gradual emergence of song selectivity in sensorimotor structures of the male zebra finch song system. J Neurosci 19, 5108-5118 (1999).

111. Amin, N., Grace, J. A. \& Theunissen, F. E. Neural response to bird's own song and tutor song in the zebra finch field L and caudal mesopallium. J Comp Physiol A Neuroethol Sens Neural Behav Physiol 190, 469-489, https://doi.org/10.1007/s00359-004-0511-x (2004).

112. Moorman, S. \& Nicol, A. U. Memory-related brain lateralisation in birds and humans. Neurosci Biobehav Rev, https://doi. org/10.1016/j.neubiorev.2014.07.006 (2014).

113. De Groof, G., Balthazart, J., Cornil, C. A. \& Van der Linden, A. Topography and Lateralized Effect of Acute Aromatase Inhibition on Auditory Processing in a Seasonal Songbird. J Neurosci 37, 4243-4254, https://doi.org/10.1523/JNEUROSCI.1961-16.2017 (2017).

114. Eales, L. A. Song learning in female-raised zebra finches: another look at the sensitive period. Anim Behav 35, 1356-1365 (1987)

115. Slater, P. J. B., Jones, A. \& Tencate, C. Can Lack Of Experience Delay The End Of The Sensitive Phase For Song Learning. Neth. J. Zool. 43, 80-90 (1993).

116. Chew, S. J., Mello, C., Nottebohm, F., Jarvis, E. \& Vicario, D. S. Decrements in auditory responses to a repeated conspecific song are long-lasting and require two periods of protein synthesis in the songbird forebrain. Proc Natl Acad Sci USA 92, 3406-3410 (1995).

117. Miller-Sims, V. C. \& Bottjer, S. W. Development of neural responsivity to vocal sounds in higher level auditory cortex of songbirds. J Neurophysiol 112, 81-94, https://doi.org/10.1152/jn.00484.2013 (2014).

118. Smulders, T. V. \& Jarvis, E. D. Different mechanisms are responsible for dishabituation of electrophysiological auditory responses to a change in acoustic identity than to a change in stimulus location. Neurobiol Learn Mem 106, 163-176, https://doi.org/10.1016/j. nlm.2013.08.010 (2013)

119. Ono, S., Okanoya, K. \& Seki, Y. Hierarchical emergence of sequence sensitivity in the songbird auditory forebrain. J Comp Physiol A Neuroethol Sens Neural Behav Physiol 202, 163-183, https://doi.org/10.1007/s00359-016-1070-7 (2016).

120. Dillon, T. S., Fox, L. C., Han, C. \& Linster, C. 17 beta-Estradiol Enhances Memory Duration in the Main Olfactory Bulb in CD-1 Mice. Behavioral Neuroscience 127, 923-931, https://doi.org/10.1037/a0034839 (2013).

121. Remage-Healey, L., Krentzel, A. A., Macedo-Lima, M. \& Vahaba, D. Species Diversity Matters in Biological Research. Policy Insights from the Behavioral and Brain Sciences 4, 210-218, https://doi.org/10.1177/2372732217719908 (2017).

122. Gresack, J. E., Kerr, K. M. \& Frick, K. M. Life-long environmental enrichment differentially affects the mnemonic response to estrogen in young, middle-aged, and aged female mice. Neurobiol Learn Mem 88, 393-408 (2007).

123. Gresack, J. E., Kerr, K. M. \& Frick, K. M. Short-term environmental enrichment decreases the mnemonic response to estrogen in young, but not aged, female mice. Brain Res 1160, 91-101, https://doi.org/10.1016/j.brainres.2007.05.033 (2007).

124. Schneider, C. A., Rasband, W. S. \& Eliceiri, K. W. NIH Image to ImageJ: 25 years of image analysis. Nat Methods 9, 671-675 (2012).

125. Böhner, J. Early acquisition of song in the zebra finch, Taeniopygia guttata. Anim Behav 39, 369-374, https://doi.org/10.1016/ S0003-3472(05)80883-8 (1990).

126. Immelmann, K. In Bird Vocalizations (ed W. H. Thorpe) Ch. 4 (1969).

127. Clayton, D. F. The genomics of memory and learning in songbirds. Annual review of genomics and human genetics 14, 45-65, https://doi.org/10.1146/annurev-genom-090711-163809 (2013).

128. Funabiki, Y. \& Konishi, M. Long memory in song learning by zebra finches. J Neurosci 23, 6928-6935 (2003).

129. Braaten, R. F. Song recognition in zebra finches: Are there sensitive periods for song memorization? Learn Motiv 41, 202-212, https://doi.org/10.1016/j.lmot.2010.04.005 (2010).

130. Griffiths, R., Double, M. C., Orr, K. \& Dawson, R. J. A DNA test to sex most birds. Mol Ecol 7, 1071-1075 (1998). 
131. Saldanha, C. J., Schlinger, B. A., Micevych, P. E. \& Horvath, T. L. Presynaptic N-methyl-D-aspartate receptor expression is increased by estrogen in an aromatase-rich area of the songbird hippocampus. J Comp Neurol 469, 522-534, https://doi. org/10.1002/cne.11035 (2004).

132. Ikeda, M., Rensel, M. A., Schlinger, B. A. \& Remage-Healey, L. In vivo detection of fluctuating brain steroid levels in zebra finches. Cold Spring Harb Protoc 2014, 1267-1272, https://doi.org/10.1101/pdb.prot084616 (2014).

133. Wynne, R. D., Walters, B. J., Bailey, D. J. \& Saldanha, C. J. Inhibition of injury-induced glial aromatase reveals a wave of secondary degeneration in the songbird brain. Glia 56, 97-105, https://doi.org/10.1002/glia.20594 (2008).

134. Balthazart, J. \& Ball, G. F. Brain aromatase, estrogens, and behavior. (Oxford University Press, 2013).

135. Peterson, R. S., Lee, D. W., Fernando, G. \& Schlinger, B. A. Radial glia express aromatase in the injured zebra finch brain. J Comp Neurol 475, 261-269, https://doi.org/10.1002/cne.20157 (2004).

136. Saldanha, C. J., Burstein, S. R. \& Duncan, K. A. Induced synthesis of oestrogens by glia in the songbird brain. J Neuroendocrinol 25, 1032-1038, https://doi.org/10.1111/jne.12067 (2013).

137. Kojima, S. \& Doupe, A. J. Social performance reveals unexpected vocal competency in young songbirds. Proc Natl Acad Sci USA 108, 1687-1692, https://doi.org/10.1073/pnas.1010502108 (2011).

138. King, A. P., West, M. J. \& Goldstein, M. H. Non-vocal shaping of avian song development: Parallels to human speech development. Ethology 111, 101-117, https://doi.org/10.1111/j.1439-0310.2004.01039.x (2005).

139. Marler, P. \& Peters, S. Sensitive Periods for Song Acquisition from Tape Recordings and Live Tutors in the Swamp Sparrow, Melospiza-Georgiana. Ethology 77, 76-84 (1988).

140. Thorpe, W. H. The learning of song patterns by birds, with especial reference to the song of the chaffinch Fringilla coelebs. Ibis 100, 535-570, https://doi.org/10.1111/j.1474-919X.1958.tb07960.x (1958).

141. Tchernichovski, O., Mitra, P. P., Lints, T. \& Nottebohm, F. Dynamics of the vocal imitation process: how a zebra finch learns its song. Science 291, 2564-2569, https://doi.org/10.1126/science.1058522 (2001).

142. Meitzen, J., Moore, I. T., Lent, K., Brenowitz, E. A. \& Perkel, D. J. Steroid hormones act transsynaptically within the Forebrain to regulate neuronal phenotype and song stereotypy. Journal of Neuroscience 27, 12045-12057, https://doi.org/10.1523/ Jneurosci.3289-07.2007 (2007).

143. Meitzen, J., Thompson, C. K., Choi, H., Perkel, D. J. \& Brenowitz, E. A. Time course of changes in Gambel's white-crowned sparrow song behavior following transitions in breeding condition. Hormones and Behavior 55, 217-227, https://doi.org/10.1016/j. yhbeh.2008.10.006 (2009).

144. Alward, B. A., Balthazart, J. \& Ball, G. F. Differential effects of global versus local testosterone on singing behavior and its underlying neural substrate. Proc Natl Acad Sci USA 110, 19573-19578, https://doi.org/10.1073/pnas.1311371110 (2013).

145. Aronov, D., Andalman, A. S. \& Fee, M. S. A specialized forebrain circuit for vocal babbling in the juvenile songbird. Science 320, 630-634, https://doi.org/10.1126/science.1155140 (2008).

146. Tomaszycki, M. \& Adkins-Regan, E. Experimental alteration of male song quality and output affects female mate choice and pair bond formation in zebra finches. Anim Behav 70, 785-794 (2005).

147. Holveck, M.-J. \& Riebel, K. Preferred songs predict preferred males: consistency and repeatability of zebra finch females across three test contexts. Anim Behav 74, 297-309, https://doi.org/10.1016/j.anbehav.2006.08.016 (2007).

148. Blumstein, D. T. \& Daniel, J. C. Quantifying behavior the JWatcher way. (Sinauer Associates, 2007).

149. Krentzel, A. A., Macedo-Lima, M., Ikeda, M. Z. \& Remage-Healey, L. A membrane g-protein coupled estrogen receptor is necessary but not sufficient for sex-differences in zebra finch auditory coding. Endocrinology, en.2017-03102-en.02017-03102, https://doi.org/10.1210/en.2017-03102 (2018).

150. Ikeda, M. Z., Jeon, S. D., Cowell, R. A. \& Remage-Healey, L. Norepinephrine Modulates Coding of Complex Vocalizations in the Songbird Auditory Cortex Independent of Local Neuroestrogen Synthesis. J Neurosci 35, 9356-9368, https://doi.org/10.1523/ JNEUROSCI.4445-14.2015 (2015).

151. Margoliash, D. Acoustic parameters underlying the responses of song-specific neurons in the white-crowned sparrow. J Neurosci 3, 1039-1057 (1983).

152. Margoliash, D. Preference for autogenous song by auditory neurons in a song system nucleus of the white-crowned sparrow. $J$ Neurosci 6, 1643-1661 (1986).

153. Green, D. M. \& Swets, J. A. Signal detection theory and psychophysics. (John Wiley, 1966).

154. Bauer, E. E. et al. A synaptic basis for auditory-vocal integration in the songbird. J Neurosci 28, 1509-1522, https://doi.org/10.1523/ JNEUROSCI.3838-07.2008 (2008)

155. Tremere, L. A., Jeong, J. K. \& Pinaud, R. Estradiol shapes auditory processing in the adult brain by regulating inhibitory transmission and plasticity-associated gene expression. J Neurosci 29, 5949-5963, https://doi.org/10.1523/JNEUROSCI.0774-09.2009 (2009).

156. R: A language and environment for statistical computing. $R$ Foundation for Statistical Computing ( $R$ Foundation for Statistical Computing, Vienna, Austria, 2018).

157. RStudio: Integrated Development for R. (RStudio, Inc., Boston, MA, 2016).

\section{Acknowledgements}

From the Healey Lab, we thank Drs. Maaya Ikeda, Ben Pawlisch, and Catherine de Bournoville for help with microdialysis; Matheus Macedo-Lima assistance with electrophysiology analysis, immunocytochemistry, and subsequent imaging; Olivia Li and Alex Rizzo for help with histology; and Christina Moschetto for quantifying female phonotaxis behavior. We also thank Dr. Colin Saldanha (American University) for the aromatase antibody; Dr. James Chambers for imaging assistance (UMass Amherst/Light Microscopy Core); and Drs. David Vicario and Mimi Phan (Rutgers University) for advice on analyzing habituation physiology data. This work was supported by the National Science Foundation IOS 1354906 (LRH) and a University of Massachusetts Amherst Graduate Student Dissertation Grant (DMV).

\section{Author contributions}

D.V. and L.R.H. conceived of the experiments. L.R.H. acquired funds for the study. D.V. and L.R.H. carried out the experiments. D.V., A.H. and L.R.H. analyzed the data. D.V. and L.R.H. wrote the main manuscript text and prepared all the figures.

\section{Competing interests}

The authors declare no competing interests.

\section{Additional information}

Supplementary information is available for this paper at https://doi.org/10.1038/s41598-020-60329-3. 
Correspondence and requests for materials should be addressed to D.M.V. or L.R.-H.

Reprints and permissions information is available at www.nature.com/reprints.

Publisher's note Springer Nature remains neutral with regard to jurisdictional claims in published maps and institutional affiliations.

(1) Open Access This article is licensed under a Creative Commons Attribution 4.0 International License, which permits use, sharing, adaptation, distribution and reproduction in any medium or format, as long as you give appropriate credit to the original author(s) and the source, provide a link to the Creative Commons license, and indicate if changes were made. The images or other third party material in this article are included in the article's Creative Commons license, unless indicated otherwise in a credit line to the material. If material is not included in the article's Creative Commons license and your intended use is not permitted by statutory regulation or exceeds the permitted use, you will need to obtain permission directly from the copyright holder. To view a copy of this license, visit http://creativecommons.org/licenses/by/4.0/.

(C) The Author(s) 2020 